\title{
A Safety Enhancement Broadcasting Scheme Based on Context Sensing in VANETs
}

\author{
Chen Chen, Hongyu Xiang, Canding Sun, and Qingqi Pei \\ State Key Laboratory of Integrated Services Networks, Xidian University, Xian 710071, China \\ Correspondence should be addressed to Chen Chen; cc2000@mail.xidian.edu.cn
}

Received 14 April 2016; Revised 3 July 2016; Accepted 4 August 2016

Academic Editor: Carlos T. Calafate

Copyright (c) 2016 Chen Chen et al. This is an open access article distributed under the Creative Commons Attribution License, which permits unrestricted use, distribution, and reproduction in any medium, provided the original work is properly cited.

\begin{abstract}
The broadcasting plays a vital role for context awareness in VANETs (Vehicular Ad Hoc Networks) whose primary goal is to improve the driving safety depending on effective information exchanging. In this paper, based on the LQG (linear quadratic Gaussian) optimal control theory, a broadcasting control scheme named LQG-CCA is proposed to improve the network throughput thus increasing the opportunities for the safety-related events to be successfully handled. By predicting the network throughput with the Kalman filter model, our LQG model is envisioned to minimize the difference between the predicted and expected throughput through the adjustment of CCA (Clear Channel Assessment) sensing threshold. Numerical results show that our proposed model can significantly improve the network performance in terms of average throughput, average End-to-End delay, and average packets delivery ratio compared with a highly cited work D-FPAV and a latest published model APPR.
\end{abstract}

\section{Introduction}

VANETs are being developed for applications on road including mainly safety-related events, such as Cooperative Collision Warning (CCW) [1], traffic signal violation warning, and lane change warning. Although the critical applications usually rely on event-driven messages exchange in very emergent cases, the periodical broadcasting in VANETs also plays an important role in safety enhancement and guarantee. For those safety-related applications, the correctness and upto-dateness of the messages greatly depend on the emergent broadcasts delivery ratio and periodical beacons frequency. For instance, in Cooperative Collision Warning or Cooperative Collision Avoidance (CCA) systems, the successful reception of braking notifications from neighboring vehicles directly influences the crash probability which depends on the intervehicle distance and/or packets transmission delay. An example is illustrated in Figure 1. Although the two following vehicles behind the front braking vehicle may have extra time to prepare for avoiding the potential crash with wireless broadcasting enabled, the packets collision from other beacons or event-driven messages may fail this emergency notification transmission. Another instance is shown in Figure 2. In intersections where approaching vehicles from different directions become blind spots in each other's horizon, at least one of the broadcasted messages should be received by the two potentially colliding vehicles to prevent severer accidents. In the traffic light violation warning scenario [2] as shown in Figure 3, reliable broadcastbased notification mechanism is crucial for collision avoidance before the specific vehicle approaches the danger area.

With above illustrations, it can be concluded that a highly efficient and reliable broadcasting mechanism is very necessary for VANETs to guarantee the driving safety. To make such mechanism practical in real cases, a model with lower communication overheads as well as complexity to greatly reduce the experienced delay is required. This scheme should also have the ability to avoid the traffic congestion especially for the safety-related events. Generally speaking, the primary cause of the network congestion is that the network loads from the nodes exceed the storage and processing capacity of the network. Therefore, the purpose of an effective congestion control is to reasonably control the channel loads and to ensure that the loads put on the network are 


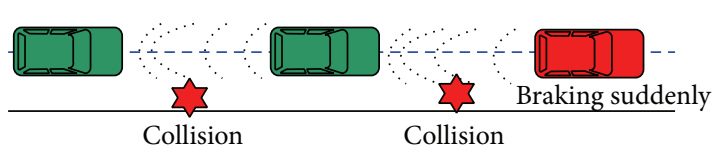

FIgure 1: Cooperative Collision Avoidance scenario.

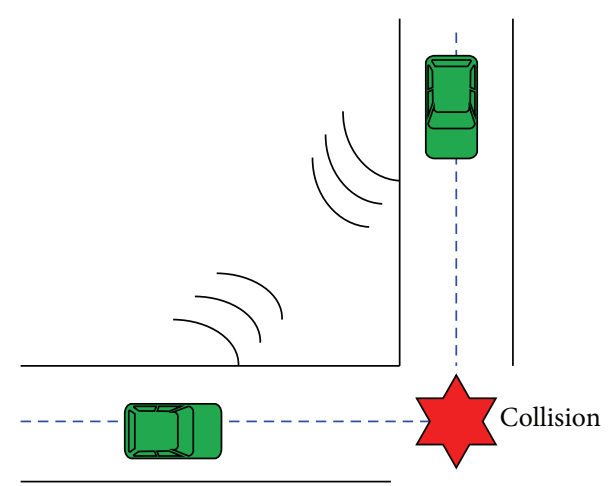

FIgURE 2: Blind spot detection scenario.

within the resource and processing capacity of the network while meantime making full use of the provided network resources, such as spectrum in our work. Fortunately, many achievements have been made on the congestion control issue in the research field of wireless Ad Hoc networks [35]. But due to the specificity of VANETs (i.e., rapid changing topology, specific mobility model, and variable vehicular density), the strategies used in the wireless Ad Hoc network cannot be directly applied to VANETs [6]. As a result, in this paper, a broadcasting congestion control framework has been proposed which takes the special characteristics of VANETs into account and attempts to fully utilize the wireless spectrum to maximize the network throughput while not making the channel congested at the same time, especially for the safety-related cases.

Note that our proposed model is adaptive to the network contexts such as traffic status and channel states through the adjusting of the CCA threshold. In this way, the nodes in a network can independently decide their channel sensing status through different local CCA configurations, thus making the global throughput maximized. In addition, by adaptively controlling the CCA threshold, the packets collisions due to hidden terminals could also be significantly alleviated [7]. Meanwhile, since we aim to control the channel congestion as well as maximize the network throughput, the requirements between transmission delay and network goodput especially for safety-related broadcasting messages could be well balanced in VANETs.

The rest of this paper is organized as follows. Section 2 describes some related works and gives their pros and cons. Section 3 provides the details of our proposed LQG-CCA model. Section 4 evaluates the performance of our model with numerical results generated via NS2. Section 5 concludes this paper.

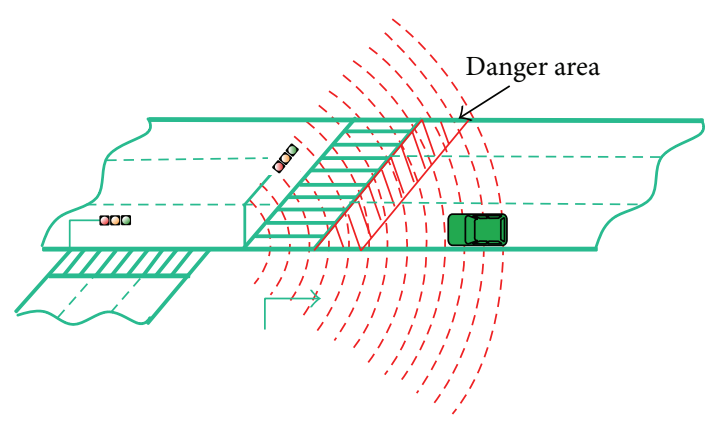

Figure 3: Traffic light violation scenario.

\section{Related Work}

By looking at the safety- or non-safety-related applications and their data traffic in VANETs, as well as the current and foreseen possible multichannel strategies, there is a risk that the corresponding radio spectrum could be readily saturated if no congestion control algorithms are taken especially in the dense environment. This channel saturation would result in unstable vehicular communications thus failing the promised applications. Therefore, although the congestion control scheme now has not been proposed as a mandatory term in related standards or drafts, we think it is extremely necessary to include this feature in the VANETs standardization process and its future realization. Considering the high mobility and dynamic network topology in VANETs, a lot of congestion control algorithms have been executed in a centralized way which has been proved to be successful. P. Rani and M. Rani [8] proposed a centralized scheduling technique by controlling congestion for safety messages in the vehicular environment. Guan et al. [9] proposed a centralized adaptive congestion control method for the vehicular networks in road intersections. Wang et al. [10] presented a method for finding an optimal tradeoff between network congestion and the freshness of received information in a cellular-based vehicular network. Although previous studies showed that centralized control might be suitable and efficient in the vehicular environment, their results are actually only applicable to the case where delay requirement is not much strict. For our discussed safetyrelated events, the introduced communication overheads from/to the central control node may be huge compared to the notifications or context aware messages between vehicles and might finally make the designed model impractical and events fail. As a result, the distributed and self-organized capability is sometime preferable in VANETs especially for delay sensitive applications.

For decentralized congestion control schemes, there have been several works which can be mainly classified into 3 categories, that is, utility, power control, and rate adjustment based. Some works also seek methods to avoid information congestion by regulating beacon frame length, but we consider this sort of strategy to be not representative because packets are generally very small in VANETs especially for safety-related notifications. 
For utility-based methods, the design principle is to assume a strictly concave utility function $u(r)$ of some given network parameters such as packet sending rate, channel capacity, transceiver power level, network throughput, or their specific combinations. The congestion control scheme is then a way to adjust the parameters to maximize total utility of the system. Wischhof and Rohling [11] proposed a Utility-Based Packet Forwarding and Congestion Control scheme (UBPFCC) that works on top of IEEE 802.11x series MAC protocols with emphasis on nonsafety applications. They designed an application-specific utility function and encoded the payoff information in each transmitted data packet from a node locally. Then, a decentralized algorithm was used to assign a share of the available data rate to nodes proportional to their relative priorities which were calculated based on their utilities to the "average utility value." However, as the authors stated in their paper, their work is only applicable to the comfort applications of VANETs whereas the safety-related applications, which have very stringent delay and reliability requirements, were not considered at all. Bouassida and Shawky [12] presented a cooperative and fully distributed congestion control approach in VANETs based on dynamic priority scheduling and transmission. Their priority is composed of two parts, that is, the static and dynamic part, and its assignment took the "messages" utility into consideration through counting the number of packets retransmissions in the neighborhood. By combining the static assignment depending on the application type and the dynamic determination upon the specific context of the VANETs (neighborhood density, node speed, and message utility and validity), the priorities of sent packets were designated and used for messages scheduling in order to avoid congestion in the overall network. However, their application type specific priority configuration is a little bit arbitrary where no metric or calculation formula is given to determine the exact value of the priority for a specific service. Additionally, how to determine "Service Channel Congestion Threshold" was not given in this paper. Zhou et al. [13] proposed a scheme jointly formulating the rate control, medium access control, and routing problem for cooperative VANETs in the framework of the utility function optimization. Although their utility function considered the tradeoff between users fairness, network cost, and users rewards, their work cannot be used in safety background where delay requirement and QoS (Quality of Service) should be taken into account.

For congestion control strategies relying on transmitting power adjustment, the design goal is to balance the tradeoff between power and packets generation rate. Generally, although a higher packet generation rate can increase the information accuracy with frequent updates, an uncontrolled strategy could also readily lead to a saturated medium. Likewise, a message sent with higher transmitting power can reach further distances, but it will also increase the level of interferences to other ongoing transmissions. Therefore, how to handle the tradeoff between power and sending rate will influence the up-to-dateness of sent messages as well as the channel congestion level. Torrent-Moreno et al. [14] proposed a fully distributed and localized power control algorithm called Distributed Fair Power Adjustment for Vehicular Networks for adaptive transmitting power adjustment which is formally proven to achieve max-min fairness. Although D-FPAV was proven effective through simulations under different radio propagation models, its major concern lies on the fairness in terms of channel busy time sensed by every node in the highway. Accordingly, D-FPAV is not applicable in urban environment for safety-related applications which care more about the successful completion ratio instead of fairness. Guo et al. [15] proposed a delay-aware and reliable broadcast protocol (DR-BP) based on transmitting power control. In their model, when emergency events occur, a local optimal relay selection mechanism is designed by which only the vehicle selected can forward warning messages. In this way, the extra redundant warning messages can be effectively restrained thus not making the network congestion and improving the delay performance for safety messages.

For generation rate control on periodic beacon messages, the design goal is to balance the tradeoff between the updating rate/interval and latency of received messages in order to avoid congestion. Mitra and Mondal [16] proposed two distributed channel congestion control algorithms by controlling the message generation rate in VANETs. Note that RSUs were actually introduced in this paper for access authentication and further congestion reduction which may make this work categorized as a centralized model. However, their proposed two algorithms for message generation rate control are fully distributed and can be locally executed on each vehicle. In their first approach APPR-1, the channel load is maintained to an estimated initial value calculated depending upon the message generation rate of different vehicles. For the second approach APPR-2, the channel load is continuously increased till the percentage of message loss lies below a predefined threshold. Sommer et al. [17] proposed a novel approach to dynamic beaconing which can provide low-latency communication (i.e., very short beaconing intervals), while ensuring not to overload the wireless channel, in the presence of radio signal obstructions caused by other vehicles and by buildings. It is worth noting that their designed dynamic beaconing (DynB) scheme allows more aggressive channel use in time-variant signal shadowing environment than in realistic case where no obstacle is considered. Although DynB introduces more practical propagation models into the design process of the information dissemination schemes in vehicular networks, their major concern actually does not focus on the safety applications along with their various safety requirements. Nevertheless, their evaluation of the impact of vehicles and buildings shadowing on the performance of beaconing protocols is really beneficial. In our work, we also take the obstacles effect into account and discuss our congestion control problem in a radio signal shadowing environment. Bai et al. [18] proposed a distributed beacon scheduling scheme CABS (Context Awareness Beacon Scheduling) which is based on the spatial context information to dynamically schedule the beacon by means of a TDMA-like manner. Their numerical results showed that CABS can efficiently use the limited network resources without leading to congestion and satisfy the requirements of safety applications as well. Although their 
work also introduces the time-slot-based scheduling, CABS is actually a TDMA-like protocol where channel contention has been resolved via slots assignment by the centralized management node. However, our work attempts to model the channel competitions in a full distributed environment where different vehicles randomly access the channel locally. Sahoo et al. [19] proposed a congestion-controlled-coordinatorbased MAC (CCC-MAC) using time-slot-based medium access protocol to address the packets congestion and safety guarantee considerations in VANETs. By incorporating a pulse-based slot reservation mechanism, their proposal can ensure fast and reliable propagation of emergency messages over multiple hops under different vehicular densities. Even though CCC-MAC is confirmed by its ability to deliver safety-critical messages to around $95 \%$ of the intended recipients, it also needs centralized scheduling to ensure that all vehicles in a segment can receive time slots for their beacon transmissions. Chaabouni et al. [20] proposed a congestion control approach that uses the number of detected collisions as a metric to control the beacon generation frequency and therefore reduce the effect of congestion and improve e-Safety. Their works verified that they can achieve a balanced tradeoff between beacon information accuracy and beacon related overhead. However, in their work, the beacon generation rate is dynamically adjusted based on the number of collisions locally detected by each node, which may imply a misjudgment of the network congestion status since local information will not always correctly respond to the global state. In addition, how to determine the important threshold $V_{\text {thresh }}$ used for initiating the rate adjustment process was not given. Tielert et al. [21] designed a protocol named PULSAR (Periodically Updated Load Sensitive Adaptive Rate control) which aims at controlling channel load and in the meantime satisfying safety applications' awareness requirements. Their reactive congestion control strategy adjusts a vehicle's transmission rate based on guidance by the application layer on transmission range as well as minimum and maximum transmission rate. Although PULSAR is a distributed algorithm and can accommodate different $\mathrm{min} / \mathrm{max}$ transmission rate intervals and radio ranges, it mainly focused on access fairness but gave a little bit of consideration on differentiated traffic which was common in VANETs with different prioritized services. Javed and Khan [22] proposed a spacedivision multiple access (SDMA) technique combined with an adaptive rate control mechanism to improve the efficiency of BSM (Basic Safety Message) transmissions. Their SDMA can reduce the interference among vehicles and address the hidden-node problem by introducing space-division access opportunities. However, the efficiency of SDMA is highly dependent on the accuracy of the estimation of density, which is usually difficult to be collected on the fly in real time and be precise enough in a highly dynamic environment.

Actually, the congestion avoidance or control according to CCA threshold adjustment could be generalized to the power control category since such adjustment will directly influence the determination of the channel busy states based on carrier sense range modification, which is usually altered by the transmitting power. For the CCA threshold adjustment based on adaptive schemes, there are already many previous existent works. Zeng et al. [23] designed an EWM (emergency warning message) dissemination algorithm that uses a CCA threshold ladder setting mechanism to deal with the road vehicle's abnormal event. In this paper, message ranking is combined with CCA adjusting which is judged by Dissemination Successful Rate (DSR) and Packet Delivery Delay (PDD). However, this method is not valid for networks with IEEE 802.11p radios. Meanwhile, systematic evaluation of this conjecture is not given in the paper. Similarly, Han et al. [24] proposed a CCA threshold selection method to imitate the behaviors of IEEE 802.11 MAC in multihop networks. By calculating the interference and transmitting probability of senders, the performance of EWMs can be improved through CCA adjustment to ensure both real time and reliability of EWM transmission. However, their experiments are only tested on a stationary test bed without mobility considered, which may suffer performance degradation when mobility model is enabled. Cho et al. [25] proposed a method to set the carrier sensing threshold by computing the self-interference at a secondary user based on the distance separation in a cognitive radio oriented wireless network. In their work, the carrier sensing threshold is presented as a common parameter to control the activity of the secondary network. The proposed method has low complexity and makes it possible to compute the carrier sensing threshold in real time. However, since cognitive radio is introduced in this work, the complexity in terms of time and computation on "spectrum hole" detection and awareness is nontrivial. Schmidt et al. [26] also proposed a stepwise CCA Threshold Adaptation (CTA) scheme depending on how long a packet has been waiting for medium access. Numerical results showed that their approach can mitigate significantly the problem of local message drops and hence local congestion.

Different from the aforementioned CCA-based works, by introducing LQG optimization control model, our algorithm aims at maximizing the global throughput by controlling the CCA threshold for each node locally. In addition, through the distributed transmission opportunities control, all nodes in a network could cooperatively schedule their transmitting thus maximizing the global profit.

\section{LQG-CCA}

In this section, the LQG optimization algorithms used to control our broadcasting scheme are given in the vehicular environment. The Linear Quadratic Regulator (LQR) [27] targets the linear system that takes the form of the state space in the modern control theory, and its objective function is a quadratic function of the object's status and the control input. In LQR, a state feedback controller $S$ is designed to minimize the quadratic objective function, where $S$ is uniquely determined by the weight matrices $Q$ and $R$. Although the LQR theory is the oldest method for designing the state space, it is capable of providing the optimized control rule according to the linear feedback on the state, which is very helpful to our discussed problem.

Since high mobility inevitably introduces random disturbance into the practical vehicular network, our congestion control issue can be transformed to an optimal control 
problem to maximize the network throughput in the presence of the random disturbance. Note that we did not take the latency as our optimization objective since different services have different delay requirements and a global minimization of delay might not guarantee the success even for the most emergent event. In addition, the random disturbance is assumed to be the Gaussian noise in our work for the sake of analysis. Therefore, our control problem can then be formulated as an LQG optimal control issue. Fortunately, a famous law of segregation [28] already exists for our discussed case, enabling us to study noise reduction and congestion control separately. In this way, while we study the congestion control problem, it can be assumed that the network is free of noise; thus, all state variables could be accurately obtained.

Our algorithm mainly consists of two parts, that is, the Kalman filter model based prediction and LQG optimization congestion control. Let us discuss them one by one in the following.

3.1. Constructing the LQG Model for Our Congestion Control Problem. Actually, our congestion control problem could be formulated as a feedback control issue in the domain of the Networked Control Systems (NCSs) [29], in which the performance of a system is iteratively adjusted in a closed-loop manner according to the generated feedback from the system. In addition, as commented in almost all the literatures on NCSs, the motivations to construct such a control system via networks are its low installation and maintenance costs, high reliability, increased system flexibility, and decreased wiring. On the other hand, a networked system also introduced some other issues to influence the output performance of a system such as uncertain delay caused by traffic congestion, packets loss due to medium collision or channel failure, and throughput degradation due to limited bandwidth. In this paper, to alleviate the impact of traffic congestion on the performance of VANET broadcasting, an LQG-based optimal control model is applied to maximize the system throughput according to the measured throughput and distributed CCA threshold control.

Generally, an LQG controlled system could be further divided into the state estimation and control part [30], respectively. In our work, the state of the next interval is estimated using the Kalman filter model according to the presently measured state considering the disturbance from the system noise. Note that the packets transmissions on the shared wireless medium are vulnerable to the channel failure and packets collisions as well as noise interference. Therefore, we compensate this performance loss in advance by intelligently adjusting the coefficients of the observed state and outputted control vectors. In addition, during the control procedure, to minimize the output error between the predicted and expected throughput, our throughput maximization problem is transformed to an optimal control issue, which in return reduced the packet loss ratio and improved the system throughput.

The notations used for constructing and updating the state space model are listed at the end of the paper for convenience.

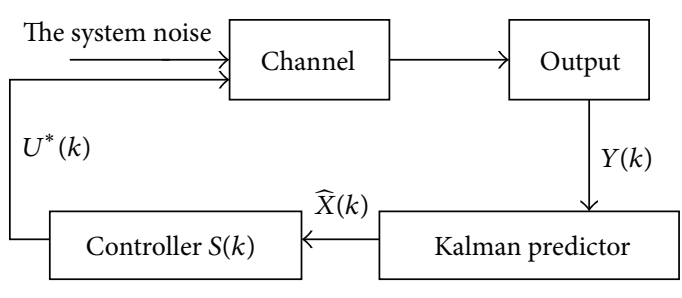

FIGURE 4: Network configuration.

Next, let us propose our LQG model regarding our congestion control problem in detail. With reference to literature [31], a state space could be described with the following discrete linear time-invariant stochastic system:

$$
\begin{aligned}
X(k+1) & =A X(k)+B U(k)+G W(k), \\
Y(k) & =C X(k)+V(k),
\end{aligned}
$$

where (1) and (2) are the state and observation equations, respectively. $X(k)$ denotes the state variable (i.e., network throughput), $U(k)$ denotes the input control (i.e., CCA threshold), and $Y(k)$ denotes the measured output. $A$ and $B$ are the coefficient matrix of the state equation. $C$ is the coefficient matrix of the observation equation. $W(k)$ and $V(k)$ are two uncorrelated white additive Gaussian noises with variances $Q(k)$ and $R(k)$, which will be discussed in detail later. $G$ denotes the gain of the Kalman filter.

The network configuration for our throughput maxmization problem through dynamic CCA adjustment could be framed as shown in Figure 4. The detailed description regarding each component is given as follows.

3.1.1. Channel. The component "channel" is used here to model the transmisson medium in a VANET. The feedback control vector is taken as the input and the measured througput is taken as the output of the channel, respectively.

3.1.2. Kalman Predictor. A "Kalman predictor" is an estimator for the linear quadratic Gaussian (LQG) problem, which is the problem of estimating the instantaneous "state" of a linear dynamic system by using measurements linearly related to the state but corrupted by Gaussian white noise. For a dynamic system, it is not always possible or desirable to measure every variable that one wants to control. The Kalman filter provides a means for inferring the missing information from indirect (and noisy) measurements. In such situations, the Kalman filter is used to estimate the complete state vector from partial state measurements and is called an observer. The resulting estimator is statistically optimal with respect to any quadratic function of estimation error.

3.1.3. Controller. The "controller" module generates the control signal by minimizing the error between the predicted and exptected state. Specifically, given a positive time horizon, the 
aim is to find the optimal control policy $\left(U^{*}\right)=\left\{U_{1}^{*}, \ldots, U_{M}^{*}\right\}$, to minimize the linear quadratic cost functional:

$$
\begin{aligned}
& J(U(k)) \\
& \quad=E\left(\sum e^{T}(k) Q(k) e(k)+U^{T}(k) R(k) U(k)\right),
\end{aligned}
$$

where $J(U(k))$ is the cost function, $e(k)$ denotes the deviation of the predicted value from the expectation, $U(k)$ is the control variable, and the matrices $Q(k)$ and $R(k)$ are, respectively, the weighting matrices indicating the state and control cost penalties. $Q(k)$ and $R(k)$ are determined through multiple numerical tests in our work which will be discussed later.

By minimizing (3), the optimal control vector could be given as follows:

$$
U^{*}(k)=-S(k) \widehat{X(k)},
$$

where $S(k)$ is the coefficient matrix, $\widehat{X(k)}$ is the input, and $U^{*}(k)$ is the output of the optimal controller, respectively.

\subsection{Modeling the Relationship between the CCA Threshold} and Throughput. To construct our LQG model and solve the congestion problem according to effective CCA control, the relationship between the CCA and finalized throughput should be figured out at first.

As the de facto standard of VANETs, the IEEE 802.11p [32] enables the CSMA/CA scheme to coordinate nodal transmission of messages. The CSMA/CA specifies that the node first senses the channel before transmitting, and it only sends the data when it senses that the channel is idle. If it finds that the channel is busy, which means another node is occupying the channel, the node should back off for a random duration for retransmission. For the CSMA/CA mechanism, the CCA threshold actually corresponds to a carrier sense area, with the node's sensing range being in reverse proportion to the CCA threshold. In other words, a higher CCA means that the node's sense area is smaller, resulting in an increased probability that the node attempts to transmit, even if another node is already transmitting on this channel. If the CCA is reduced, then the node's sensing range will instead increase, making it more likely to sense the channel being busy. As a result, the node will have a lower probability of trying to transmit. In conclusion, the higher the CCA threshold, the higher the collision probability. In addition, with the dropping of the CCA threshold, not only the collision probability but also the throughput and channel utilization decline.

In fact, the network capacity is an important measure of the network performance which mainly depends on two indexes, that is, the channel throughput and spatial multiplexing level. In general, the channel throughput refers to the amount of data transmitted on the channel per unit time, and it mainly relies on the SINR (Signal to Interference and Noise Ratio) of the current channel. The spatial multiplexing level indicates the number of node pairs that can transmit in parallel across the network and it is determined by the transmission power and CCA threshold. In our work, by adjusting the CCA threshold, some nodes will be restrained from transmitting thus increasing the opportunities of concurrent transmissions for others, improving the network throughput to a great extent through spatial multiplexing.

Next, according to the framework in Figure 4, our congestion control problem could be further formulated in detail as follows. As defined in (1) and (2), $X(k)$ denotes the network throughput and $U(k)$ denotes the CCA threshold in our work, respectively. We predict the throughput and compare it with the expected one, that is, the optimal throughput, and minimize the difference between the predicted and expected throughput by controlling the CCA threshold using our presented LQG controller. Actually, by adjusting the CCA threshold, some nodes will be restrained from transmitting thus increasing the opportunities of concurrent transmissions for others and improving the network throughput to a great extent.

As a result, before constructing the LQG model, we should first derive the relationship between the CCA threshold and throughput. The notations mentioned later to deduce their relationship are listed at the end of the paper for convenience.

In our envisioned model, a dense network is assumed and wireless stations are uniformly and independently distributed in an area of Area. A common and fixed transmission power $P$ is used by each transmitter.

Assume that a vehicle produced an emergency warning message (EWM). Let us define the interferences to this source node within the investigated area Area:

$$
I_{s}=\sum_{m \in \text { Area }} \sum_{i \in \text { Area }} \pi_{m} \text { Pow }(i, k),
$$

where $\pi_{m}$ is a constant. $\operatorname{Pow}(i, k)$ is the signal strength on the receiver for packets from $i$ at time $k$; that is,

$$
\begin{aligned}
\text { Pow } & (i, k) \\
= & \text { Pow }(i) \\
& -\left[92.44+20 \log f(\mathrm{GHZ})+\log d_{i k}(\mathrm{~km})\right],
\end{aligned}
$$

where $d_{i k}$ indicates the distance between $i$ and its intended receiver at time $k$. Pow $(i)$ is the transmitting power of $i$.

To make the EWM successfully decoded, the SINR of the received signal should at least meet the SINR threshold at the receiver, say, tolerate an interference no smaller than $I_{s}$. With the CCA definition, we have

$$
\mathrm{CCA}_{\mathrm{th}} \geq I_{s} .
$$

As a result, to make the throughput maximized, the CCA will be

$$
\mathrm{CCA}_{\mathrm{th}}=I_{s}
$$

Correspondingly, the SINR for this case can be expressed as follows:

$$
\operatorname{SINR}=\frac{\text { Pow }(i, k)}{N_{0}+I_{s}}=\frac{\text { Pow }(i, k)}{N_{0}+\mathrm{CCA}_{\mathrm{th}}},
$$

where $N_{0}$ is the noise power. 


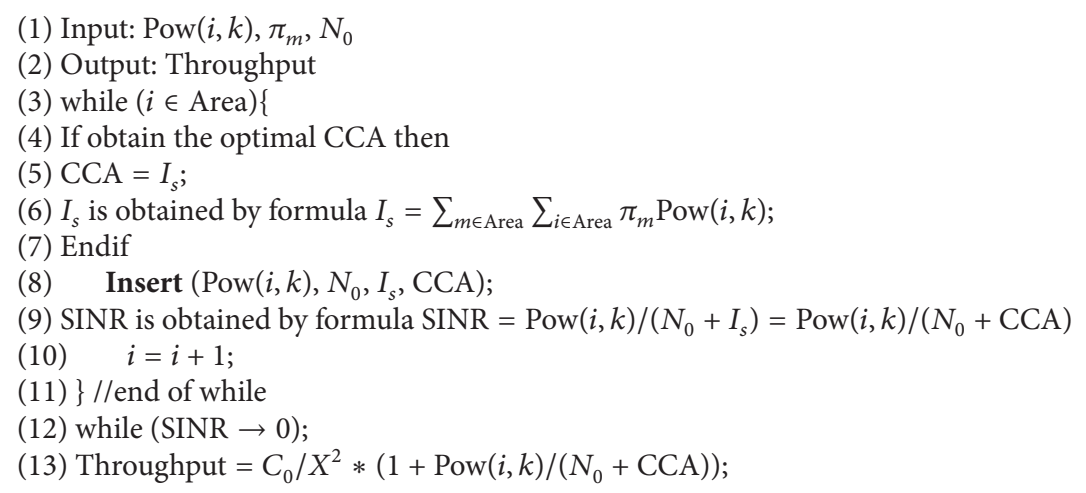

Algorithm 1: The derivation of the relation between throughput and CCA.

With the above analysis, the relation between throughput and CCA could be given as follows [33]:

$$
\begin{aligned}
\text { Throughput } & =\text { ChannelRate } * M \\
& =C_{0} * \frac{\ln (1+\mathrm{SINR})}{X^{2}},
\end{aligned}
$$

where ChannelRate is the achievable channel rate expressed with Shannon capacity; that is, ChannelRate $=W \log _{2}(1+$ SINR). $W$ is the channel bandwidth in Hertz. $M$ accounts for the total number of concurrent transmissions. $C_{0}$ is a constant. $X=D / R$, where $D$ is the carrier sense range and $R$ denotes the maximum radio range.

When SINR $\rightarrow$, throughput can be simplified as follows:

$$
\text { Throughput }=\frac{C_{0}}{X^{2}} *\left(1+\frac{\text { Pow }(i, k)}{N_{0}+\mathrm{CCA}}\right) .
$$

The pseudocode used to deduce the relationship between throughput and CCA can be described as shown in Algorithm 1 .

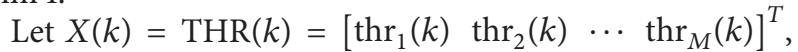
where $\operatorname{thr}_{i}(k)$ is the throughput of vehicle $i$ at time $k$. Then, $\operatorname{thr}_{i}(k)$ can be derived as follows:

$$
\operatorname{thr}_{i}(k)=\frac{C_{0}}{X_{i}^{2}} *\left(1+\frac{\operatorname{Pow}(i, k)}{N_{0}+\mathrm{CCA}_{i}}\right) .
$$

Let $U(k)=\left[\begin{array}{lllll}\mathrm{CCA}_{1}(k) & \mathrm{CCA}_{2}(k) & \cdots & \mathrm{CCA}_{M}(k)\end{array}\right]^{T}$, where $\mathrm{CCA}_{i}$ is the CCA of vehicle $i$, and then (1) can be further derived as follows:

$$
\begin{array}{r}
\operatorname{THR}(k+1)=A * \operatorname{THR}(k)+B * \operatorname{CCA}(k) \\
+G W(k)=\left[\begin{array}{c}
\operatorname{thr}_{1}(k+1) \\
\operatorname{thr}_{2}(k+1) \\
\vdots \\
\operatorname{thr}_{M}(k+1)
\end{array}\right]
\end{array}
$$$$
=A\left[\begin{array}{c}
\operatorname{thr}_{1}(k) \\
\operatorname{thr}_{2}(k) \\
\vdots \\
\operatorname{thr}_{M}(k)
\end{array}\right]+B\left[\begin{array}{c}
\operatorname{CCA}_{1}(k) \\
\operatorname{CCA}_{2}(k) \\
\vdots \\
\operatorname{CCA}_{M}(k)
\end{array}\right]
$$$$
+G\left[\begin{array}{c}
w_{1} \\
w_{2} \\
\vdots \\
w_{M}
\end{array}\right]
$$

Since nodes share the wireless medium, the throughput of each node in the network is dependent on each other. Therefore, the state transition matrix $A$ could be calculated as follows.

For an $M$-node network, the Markov chain is characterized by the $M \times M$ transition matrix $A=\left[p_{n k}\right]$ with $p_{n k}$ being the probability that the network state goes from $n$ to $k$ in one transition. By [34], the Markov chain transition matrix $A$ can be obtained as $A=\left[p_{n k}\right]$, where

$$
\begin{aligned}
& p_{n k} \\
& =\left\{\begin{array}{l}
\sum_{y=n-k}^{n} \sum_{x=0}^{M-n} r_{(x+y)[x+(n-k)]} Q_{r}(y, n) Q_{t}(x, n), \quad 0 \leq k \leq n \\
\sum_{x=k-n}^{M-n} \sum_{y=0}^{n} r_{(x+y)[x-(k-n)]} Q_{t}(y, n) Q_{r}(y, n), \quad n \leq k \leq M,
\end{array}\right. \\
& Q_{t}(k, n)=\left(\begin{array}{c}
M-n \\
k
\end{array}\right)\left(1-p_{t}\right)^{M-n-k} p_{t}^{k}, \\
& Q_{r}(k, n)=\left(\begin{array}{c}
n \\
k
\end{array}\right)\left(1-p_{r}\right)^{n-k} p_{t}^{k},
\end{aligned}
$$

where $Q_{t}(k, n)$ denotes the probability of $k$ transmissions from successful nodes given that there are $n$ backlogged nodes. $Q_{r}(k, n)$ denotes the probability of $k$ transmissions from backlogged nodes given that there are $n$ backlogged 
nodes. $p_{t}$ and $p_{r}$ denote the packet transmission probability of successful and backlogged nodes.

Then, we can obtain the expression of the control coefficient $B$ in (1) as follows:

$$
B=\frac{\operatorname{THR}(k+1)-A * \operatorname{THR}(k)-G W(k)}{\operatorname{CCA}(k)} .
$$

Further, take $X(k)$ and $U(k)$ into (15) and simplify it; we can get the expression of $B$ as

$$
\begin{aligned}
& B=\left[\begin{array}{llll}
B_{1}(k) & B_{2}(k) & \cdots & B_{M}(k)
\end{array}\right]^{T}, \\
& B_{i}(k)=\frac{C_{0}}{X_{i}^{2} * \operatorname{CCA}(k)} \cdot[\operatorname{Pow}(i, k) \\
& \left.*\left(\frac{1}{N_{0}+\operatorname{CCA}(k+1)}-\frac{\sum_{j=1}^{M} p_{1 j}}{N_{0}+\operatorname{CCA}(k)}\right)\right] \\
& -\frac{C_{0}}{X_{i}^{2} * \operatorname{CCA}(k)} \cdot\left(\sum_{j=1}^{M} p_{1 j}-G_{1} W_{1}(k)\right),
\end{aligned}
$$

where $\sum_{j=1}^{M} p_{1 j}$ is the sum of the first row of matrix $A$.

In [35], a strategy for dynamic iterative Kalman filtering has been proposed which proved that the output of every node in a Kalman filter is independent of each other. According to this conclusion, we assume the $M$ nodes have identical measurement coefficient; that is, $C_{1}(k)=C_{2}(k)=\cdots=$ $C_{M}(k)=1$. Then, we could thereby take the measurement coefficient $C(k)=I$. Further, for the noise variables $W(k)$ and $V(k)$ in (1) and (2), they are two uncorrelated white Gaussian noises with covariances $Q(k)$ and $R(k)$; that is, they are independent of each other. Meanwhile, in $W(k)=$ $\left[\begin{array}{llll}w_{1} & w_{2} & \cdots & w_{M}\end{array}\right], w_{i}(i=1,2, \ldots, M)$ is distributed as a multivariate Gaussian with expectation 0 and covariance $Q(k)$ and, in $V(k)=\left[\begin{array}{llll}v_{1} & v_{2} & \cdots & v_{M}\end{array}\right], v_{i}(i=1,2, \ldots, M)$ is distributed as a multivariate Gaussian with expectation 0 and covariance $R(k)$.

Then all variables in (1) and (2) have been deduced now.

3.3. Optimal Estimation. To accurately estimate the network throughput in the next discrete interval, the Kalman filter model is introduced to remove the noise from the estimation. With the network throughput at previous interval and the observation of throughput on the current interval, the Kalman filter can eliminate the impact of the noise on the state variable and iteratively compute the estimation of the network throughput on the current interval. To remove the noise with the Kalman filter, two stages are usually necessary, that is, estimating and updating. At the estimating stage, the filter forecasts the network throughput of the current interval using the estimated throughput at the previous interval according to the relation between carrier sense threshold and network throughput. At the updating stage, the filter uses the observation on the network throughput of the current interval to amend the estimation of the network throughout obtained at the estimation stage, resulting in an accurate observation on the throughput.
Let $k-1$ denote the previous interval and $k$ denote the current interval, and suppose that system's network throughput and covariance at $k-1$ are known as $X(k-1 \mid k-1)$ and $Z(k-1 \mid k-1)$, respectively. Thereupon, the network throughput at $k$ can be computed as follows:

$$
X(k \mid k-1)=A X(k-1 \mid k-1)+B U(k),
$$

where $X(k \mid k-1)$ is the estimated throughput the Kalman filter provides based on the network throughput at $k-1$. The covariance corresponding to $X(k \mid k-1)$ is computed as follows:

$$
Z(k \mid k-1)=A Z(k-1 \mid k-1) A^{T}+Q(k-1),
$$

where $Q(k-1)$ indicates the variance of $W(k-1)$. The $Q(k-1)$ is a specific value and it is determined through multiple numerical tests in our work which will be discussed in Section 3.4.

The Kalman gain $G(k)$ at $k$ is computed as follows:

$$
G(k)=\frac{Z(k \mid k-1) C^{T}}{C Z(k \mid k-1) C^{T}+R(k)},
$$

where $R$ denotes the covariance of the measurement noise $V$. With the parameters obtained, we can correct the estimation of network throughput $X(k \mid k-1)$ at $k$ made by Kalman filter in (10) to yield an accurate value of the throughput denoted by $\widehat{X(k)}$; that is,

$$
\begin{aligned}
\widehat{X(k)}= & X(k \mid k-1) \\
& +G(k)(Y(k)-C X(k \mid k-1)) .
\end{aligned}
$$

To enable the Kalman filter to operate iteratively, the covariance $Z(k \mid k)$ corresponding to $X(k \mid k)$ is given as follows:

$$
Z(k \mid k)=(1-C G(k)) Z(k \mid k-1) .
$$

With (17) (21), the predicted network throughput after the elimination of the noise can be worked out.

The prediction process is given as shown in Algorithm 2.

3.4. Computation of the Optimal Control Quantity. The linear quadratic optimal control model is used in our work to make the network throughput fulfill its expectation. In the case of network congestion, the network throughput will decrease and deviate from the expectation. By comparing the predicted network throughput with the expected one, we can compute the CCA threshold that enables the network throughput to meet the expectation using the linear quadratic optimal control model.

Next, according to the discussed LQG model in Section 3.1, our quadratic optimization control model is established as follows. First, we should define an appropriate expected network throughput $Y_{r}(k)$, which is dependent on the number of vehicles and road conditions. For the expected throughput, we establish a Markov chain to get the optimal throughput between the users with reference to the literature 


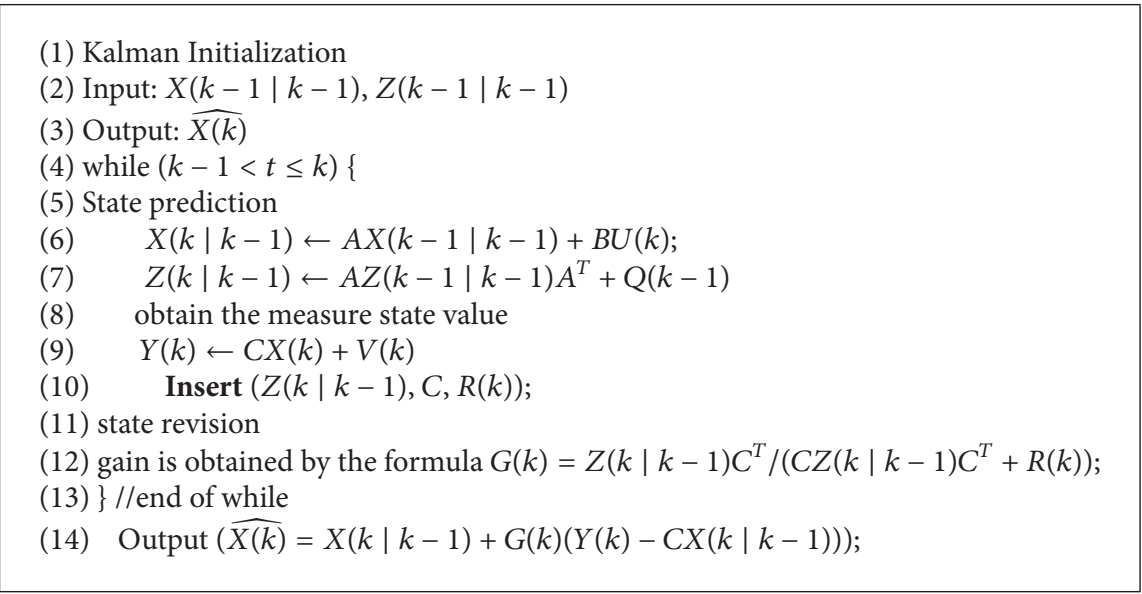

Algorithm 2: The optimal estimation.

[36]. If the network congestion occurs due to high density of vehicles, the predicted throughput $Y(k)$ will be less than $Y_{r}(k)$. Let $e(k)=Y_{r}(k)-Y(k)$ denote the deviation of the predicted throughput from the expectation. Based on this deviation, the cost function is defined as follows:

$$
J(k)=E\left(\sum e^{T}(k) Q(k) e(k)+U^{T}(k) R(k) U(k)\right),
$$

where $J(k)$ is the cost function, $E(\cdot)$ represents the mean value, $e(k)$ denotes the deviation of the predicted throughput from the expectation, $U(k)$ is the control variable, and the matrices $Q(k)$ and $R(k)$ are the weighting matrices, respectively, indicating the state and control cost penalties.

To obtain the value of $R(k)$, a numerical test is launched with different configurations of $R(k)$. According to [37], we set the order of $R(k)$ to $10^{-4}$ and choose its value as $0.1 * 10^{-4}$, $1 * 10^{-4}, 5 * 10^{-4}, 10 * 10^{-4}$, and $20 * 10^{-4}$, respectively. The corresponding results are listed in Table 1 for illustration.

As illustrated in Table 1, it can be concluded that the radio minimum is observed when

$$
R(k)=0.1 * 10^{-4} \times\left[\begin{array}{llll}
1 & 1 & \cdots & 1
\end{array}\right]_{M \times M} .
$$

$Q(k)$ indicates the variance of Gaussian noise $W(k)$. In our work, $Q(k)$ is also determined through a numerical test. At first, $Q_{0}(k)$ is initialized with the elements on its diagonal at order of $10^{-4}$ [37]; that is,

$$
Q_{0}=10^{-4} \times \operatorname{diag}\left[\begin{array}{llll}
1 & 1 & \cdots & 1
\end{array}\right]_{(M+1) \times(M+1)} .
$$

Then, we choose $Q(k)$ as $0.1 * Q_{0}, Q_{0}, 10 * Q_{0}, 100 * Q_{0}$, and $1000 * Q_{0}$ to test and the results are listed in Table 2 .

It can be noticed from Table 2 that, for the same state transition process, the bigger the $Q(k)$, the bigger the disturbance to the state variable. At the same time, when $Q(k)$ begins to increase, the speed of the convergence of $Q(k)$ becomes slow. As a result, we choose $Q(k)=0.1 * 10^{-4} \times$ $\operatorname{diag}\left[\begin{array}{llll}1 & 1 & \cdots & 1\end{array}\right]_{M \times M}$ in our work.

Using LQG benchmark, the achievable performance of $J(k)$ is given by a tradeoff curve between $\operatorname{var}(Y(k))$ and $\operatorname{var}(U(k))$ as shown in Figure 5 and this curve can be further obtained by solving the LQG problem [38].
TABLE 1: Kalman filtering error table with different matrix $R(k)$.

\begin{tabular}{lcc}
\hline $\begin{array}{l}\text { Parameters for } \\
\text { filtering } R(k)\end{array}$ & $\begin{array}{c}\text { Predicted } \\
\text { value } \overline{X(k)}\end{array}$ & $\begin{array}{c}\text { The ratio of predicted } \\
\text { value to the measured } \\
\text { value }\end{array}$ \\
\hline $0.1 * 10^{-4}$ & 30.96 & $41.25 \%$ \\
$1 * 10^{-4}$ & 23.81 & $31.72 \%$ \\
$5 * 10^{-4}$ & 34.60 & $46.10 \%$ \\
$10 * 10^{-4}$ & 53.54 & $71.34 \%$ \\
$20 * 10^{-4}$ & 93.97 & $95.20 \%$ \\
Measured value & 75.06 & $100 \%$ \\
$Y(k)$ & & \\
\hline
\end{tabular}

TABLE 2: Kalman filtering error table with different matrix $Q(k)$.

\begin{tabular}{lcc}
\hline $\begin{array}{l}\text { Parameters for } \\
\text { filtering } Q(k)\end{array}$ & $e(k)=Y_{r}(k)-Y(k)$ & $\begin{array}{c}\text { The ratio of } e(k) \\
\text { to } Y_{r}(k)\end{array}$ \\
\hline $0.1 * Q_{0}$ & 16.68 & $39.69 \%$ \\
$Q_{0}$ & 19.87 & $47.26 \%$ \\
$10 * Q_{0}$ & 25.04 & $59.57 \%$ \\
$100 * Q_{0}$ & 33.58 & $79.89 \%$ \\
$1000 * Q_{0}$ & 39.92 & $94.97 \%$ \\
Expected value & 42.04 & $100 \%$ \\
$Y_{r}(k)$ & & \\
\hline
\end{tabular}

The LQG problem is set up to control the system by keeping the output error small via small control energy. Therefore, our problem can be formulated as the problem of minimizing the cost function to work out the optimal feedback control rule $U^{*}(k)$, that is, making $Y(k)$ equal to $Y_{r}(k)$.

With reference to [39], definition 1, the LQG system is said to be stable in the mean square sense if all initial states $X(0)$ yield

$$
\lim _{k \rightarrow \infty} E\left(X(k) X(k)^{T}\right)=0
$$




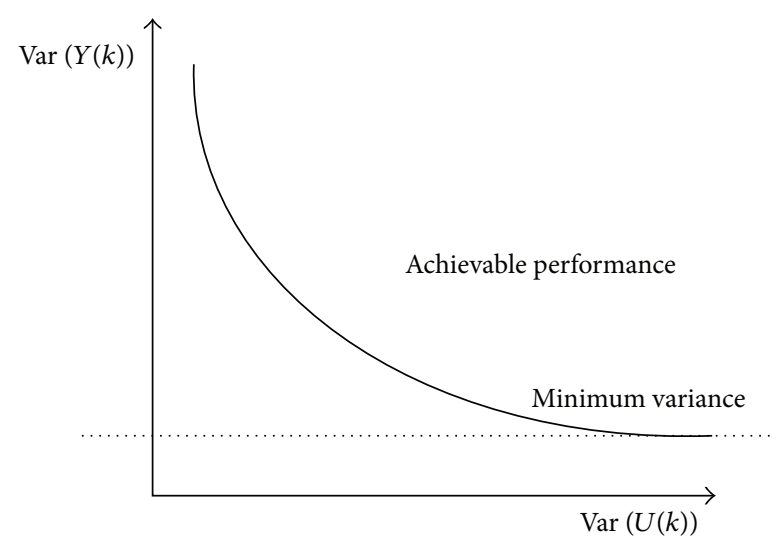

FIGURE 5: Optimal performance curve obtained through LQG benchmark.

The LQG system is said to be stabilizable in the mean square sense if there exists a matrix $S(k)$ such that

$$
\begin{aligned}
& J(U(k)) \\
& \quad=E\left(\sum e^{T}(k) Q(k) e(k)+U^{T}(k) R(k) U(k)\right)
\end{aligned}
$$

is stable in the mean square sense. We use the concept of stability here as what would in other contexts usually be called asymptotic stability. Note that stability in the mean square sense implies stability of the mean (i.e., $\lim _{k \rightarrow \infty} E\{X(k)\}=0$ ) for all $X(0)$ and almost sure stability (i.e., $\lim _{k \rightarrow \infty} X(k)=0$, for all $X(k))$. In particular, this implies that stability of the deterministic system

$$
X(k+1)=A X(k)+B U(k),
$$

is a necessary condition for mean square stability of LQG system. (This condition is satisfied if (27) is, say, controllable [40].)

Finally, according to the framework of LQG in Figure 4, our optimal controller could be expressed as (28). With reference to [38], the LQG controller computing an optimal state feedback control law by minimizing the quadratic cost function $J$ can be expressed as follows:

$$
U^{*}(k)=-S(k) \widehat{X(k)},
$$

where $\widehat{X(k)}$ denotes the predicted throughput with Kalman filtering and $S(k)$ is the optimal feedback gain matrix which can be solved via MATLAB LQ (linear quantifier) function. According to Figure 4, when the state estimation $\widehat{X(k)}$ is obtained from the Kalman filter, the optimal control vector $U^{*}(k)$ will be obtained by $\widehat{X(k)}$ multiplied by $S(k)$. The optimal state feedback gain matrix $S(k)$ is available from solving the associated discrete algebraic Riccati equation [41]; that is,

$$
\begin{array}{r}
S(k)=-\left(R(k)+B^{T} Q_{k+1}(k) B\right)^{-1} B^{T} Q_{k+1}(k) A, \\
\forall k \in\{0, \ldots, T-1\} .
\end{array}
$$

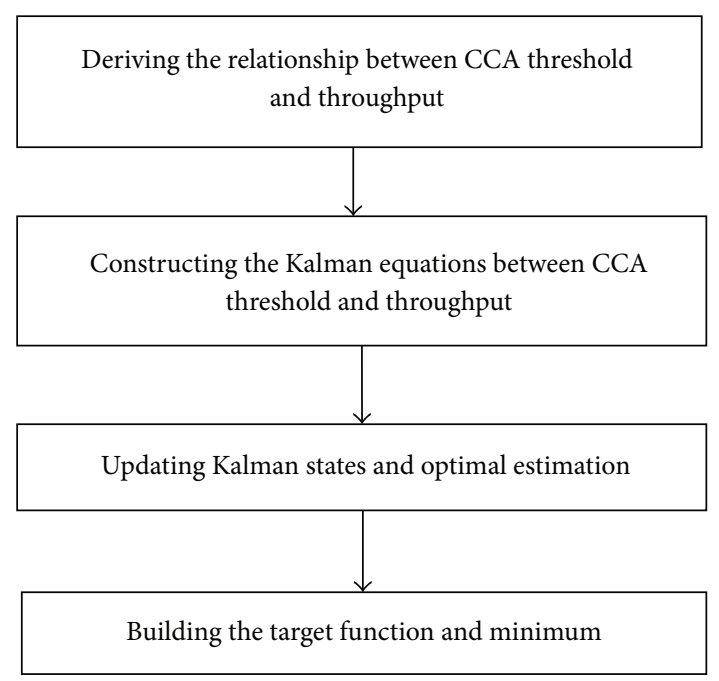

FIGURE 6: An LQG optimized information congestion control scheme.

So the corresponding minimum cost is

$$
J\left(U^{*}(k)\right)=\left[\begin{array}{c}
X(k) \\
U^{*}(k)
\end{array}\right]^{T}\left[\begin{array}{cc}
Q(k) & S(k) \\
S^{T}(k) & R(k)
\end{array}\right]\left[\begin{array}{c}
X(k) \\
U^{*}(k)
\end{array}\right] .
$$

When the optimal CCA threshold CCA $=U^{*}(k)$ is obtained, we can figure out the optimal throughput of all vehicles as follows:

$$
\text { Throughput }(k)=\sum_{i} \operatorname{thr}_{i}(k) .
$$

Finally, our proposed scheme in this paper can be summarized as a flow chart as shown in Figure 6.

\section{Simulation}

The simulation in this paper is performed in a Linux system which combines MATLAB with NS2. The topology is created with the popular Vanetmobisim toolset [42] as shown in Figure 7. The street layouts used for simulation, that is, the selected area of Washington DC, are loaded from TIGER database. The complexity of the selected area of Washington DC is 42,80 , and 125 ; that is, there are 42 junctions and 80 streets and the average length of streets is 125 meters. The labels with numbers in Figure 7(b) indicate different vehicles and they are a little bit overlapped due to limited screen space especially when they are stopped by traffic lights. The lines represent avenues or streets. Since traffic lights are enabled in simulation, the colored line means there is a traffic light on the junction. The red color line indicates that the traffic on this line is stopped by a red light and vice versa. Note that the configuration of the position and number of traffic lights are not the real case, but it can be adjusted during simulations to reflect the practical situation. In our cases, we set the number of traffic lights to 10 . In addition, although TIGER can describe land attributes such as roads, buildings, rivers, and lakes, it is still difficult to draw obstacles 
on output traces by Vanetmobisim till now. However, to reflect the influences from obstacles which are common in urban environment, we extracted the coordinates of obstacles from the investigated parts of real maps and input them into NS2. Besides, since there is no height information in TIGER database, a modification to NS2 is needed to reflect the impact of obstacles on channel fading and power attenuation. To support obstacle modeling, a two-dimension obstacle object "Obstacle Class" is introduced which represents a wall of 1 meter deep and has the length indicated by the distance between two coordinates extracted from the real maps, that is, $P_{1}\left(x_{1}, y_{1}\right)$ and $P_{2}\left(x_{2}, y_{2}\right)$. By this way, a building could be expressed by four connected walls. When the line of sight (LOS) of a communication pair intersects with the outline of the building, the power attenuation could be calculated by the following equation, which combines the generic free space path loss model with the obstacle model presented in [43]; that is,

$$
\begin{aligned}
P_{r}[\mathrm{dBm}]= & P_{t}[\mathrm{dBm}] \\
& +10 \log \left(\frac{G_{t} G_{r} \lambda^{2}}{16 \pi^{2} d^{\alpha}}\right)-\beta n-\gamma d_{m},
\end{aligned}
$$

where $P_{r}, P_{t}, G_{t}, G_{r}, \lambda, d$ are the receiving power, transmitting power, sender antenna gain, receiver antenna gain, wave length, and the distance between sender and receiver, respectively. $n$ is the number of times that the border of the obstacle is intersected by the line of sight. $d_{m}$ here is the total length of the obstacle's intersection. $\beta$ and $\gamma$ are two constants. $\beta$ is given in $\mathrm{dB}$ per wall and represents the attenuation a transmission experiences due to the (e.g., brick) exterior wall of a building. $\gamma$ is given in $\mathrm{dB}$ per meter and serves as a rough approximation of the internal structure of a building. The general values of $\beta$ and $\gamma$ in most cases are $9 \mathrm{~dB}$ and $0.4 \mathrm{~dB} / \mathrm{m}$, respectively.

The performance of our model is evaluated by varying the density, the packet generation speed, and the intervehicle distance according to the following metrics:

(1) Average throughput, defined as the average number of successfully transmitted payloads per second for the overall network.

(2) Average End-to-End delay (average E2E delay), defined as the delay averaged among all the transmitted packets by all the active vehicles during the simulation period.

(3) Average packets delivery ratio (average PDR), defined as the average ratio of the packets successfully delivered to the destinations with respect to those generated by the sources during the simulation period.

In our simulated highway scenario, all vehicles keep sending and receiving periodic beacon messages. The maximum radio range of each vehicle is set to $1,000 \mathrm{~m}$. Actually, according to the IEEE 802.11 , the fast-moving vehicles can cover a transmission range over $1,000 \mathrm{~m}$ [44]. Our scenario is envisioned comprising 2 lanes and $10 \mathrm{~km}$ long in total, where there are 50 (at least) to 400 (at most) vehicles in
TABLE 3: Simulation parameters.

\begin{tabular}{lc}
\hline Parameters & Values \\
\hline Frequency band $(\mathrm{GHz})$ & 5.92 \\
Channel data rate $(\mathrm{Mbps})$ & 3 \\
Transmission model & Nakagami \\
Maximum communication range $(\mathrm{m})$ & 1000 \\
SNR threshold for frame reception $(\mathrm{dB})$ & 5 \\
Slot time $(\mu \mathrm{s})$ & 16 \\
AIFS $(\mu \mathrm{s})$ & 9 \\
Access class & $\mathrm{AC}$ VO \\
$a C W_{\min }$ & 15 \\
$a C W_{\max }$ & 1023 \\
$C W_{\text {min }}$ (AC_VO) & $\left(a C W_{\min }+1\right) / 4-1$ \\
$C W_{\text {max }}$ (AC_VO) & $\left(a C W_{\min }+1\right) / 2-1$ \\
Beacon size $($ bytes $)$ & 500 \\
Total road length $(\mathrm{Km})$ & 10 \\
Propagation delay $(\mu \mathrm{s})$ & 1 \\
Number of traffic lights & 10 \\
Simulation time $(\mathrm{s})$ & 50 \\
\hline
\end{tabular}

TABLE 4: Scenario generation parameters for IDM_LC.

\begin{tabular}{lc}
\hline Description & Value \\
\hline Traffic light interval (s) & 3 \\
Min speed (m/s) & 6.66 \\
Recalculating movement step (s) & 1 \\
Number of lanes & 2 \\
Max speed (m/s) & 24.44 \\
Min stay (s)-max stay (s) & $5-30$ \\
\hline
\end{tabular}

two directions and the vehicle moves using the IDM_LC (intelligent driver model with lane changing) mobility model with the parameters listed in Table 4. Detailed simulation parameters are listed in Table 3. The frequency band is set to $5.92 \mathrm{GHz}$ which is configured to serve for the "High Power Public Safety" services in IEEE 802.11p. The channel data rate is chosen to be the lowest rate supported by IEEE 802.11p, namely, $3 \mathrm{Mbps}$ [45]. The Nakagami radio propagation model, whose parameters were adjusted to match actual measurements reported in [46], has been used. The Access Class is configured to AC_VO, say, the highest priority for voice transmission. This is because the packets exchanged in our discussed scenario are safety-related where the highest priority is necessary to guarantee their exclusive transmissions. The beacon size is set to 500 bytes [47]. The propagation delay is fixed to $1 \mu \mathrm{s}$ for simplicity. The simulation duration is 50 seconds and all the results are the average of 100 runs.

To evaluate the performance of our proposed LQG-CCA algorithm, two other protocols, that is, D-FPAV and APPR, are introduced for comparisons. Note that D-FPAV is a highly cited work in the vehicular communication research filed to address the channel congestion issue through transmission power control to improve the network performance in terms of dissemination delay and amount of message retransmissions. Similarly, our LQG-CCA also tends to improve the 


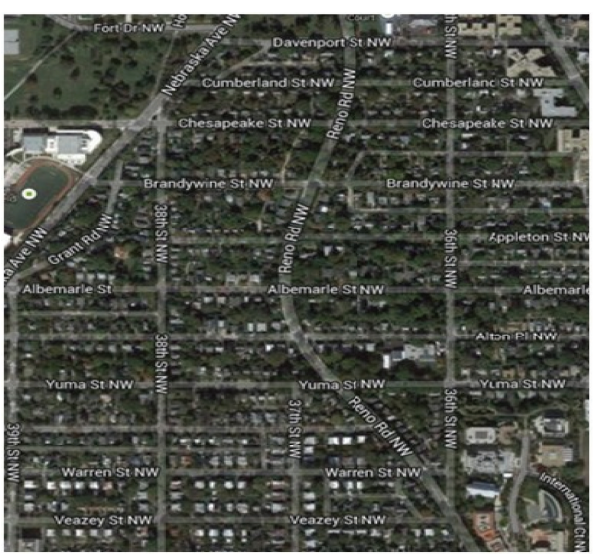

(a) Satellite snapshot

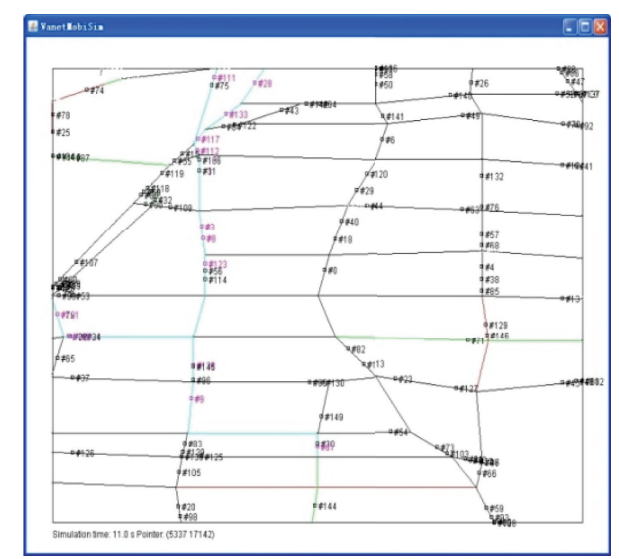

(b) Topology generated by Vanetmobisim

FIgURE 7: Simulation topology.

network performance by dynamically adjusting the CCA threshold for each node to maximize the network throughput whereas not making the channel congested. In addition, DFPAV and LQG-CCA are both designed for time-critical safety-related events in a vehicular environment. As a result, we consider D-FPAV to be a better candidate for performance comparisons with our proposed LQG-CCA. As for APPR, it is also a channel congestion reduction scheme by varying the length of beacon message, controlling the transmission power and message generation rate, and removing the duplicate messages from the message queue. Additionally, APPR is also applicable to the safety-related scenarios in which an emergency message or a warning message will be propagated according to the aforementioned congestion control strategies. Note that, in our work, only APPR_II is implemented for comparison since it is more reasonable in practice which attempts to increase the channel load till the percentage of message loss lies below a predefined threshold. Instead, APPR_I just maintains the channel load to a fixed value depending upon the message generation rate of different vehicles in a network. The important parameter in APPR_II, that is, the threshold of the message loss ratio, is set to $2 \%$ according to [16].

Figure 8 shows the network average throughput performance with the number of nodes varying from 50 to 400 . The packets generation speed for this case is 5 packets/s. It is worth noting that our LQG-CCA always outperforms the other two in this case. Before point 200, the average throughput of LQG-CCA continuously increases. And after 200 , there is a slow decrease of average throughput for LQGCCA and finally it outputs approximately $2.3 \mathrm{Mbps}$ which is even bigger than the throughput corresponding to the 50 vehicles. At first, this result indicates that the impact of the growth of senders on the throughput is larger than that of the more introduced collisions from more vehicles. This conclusion is also valid for APPR. However, D-FPAV shows a lower throughput at point 400 compared to that at point 50. The reason behind this is the transmission restraining mechanism adopted by LQG-CCA and APPR. For LQG-CCA, by dynamically computing the appropriate

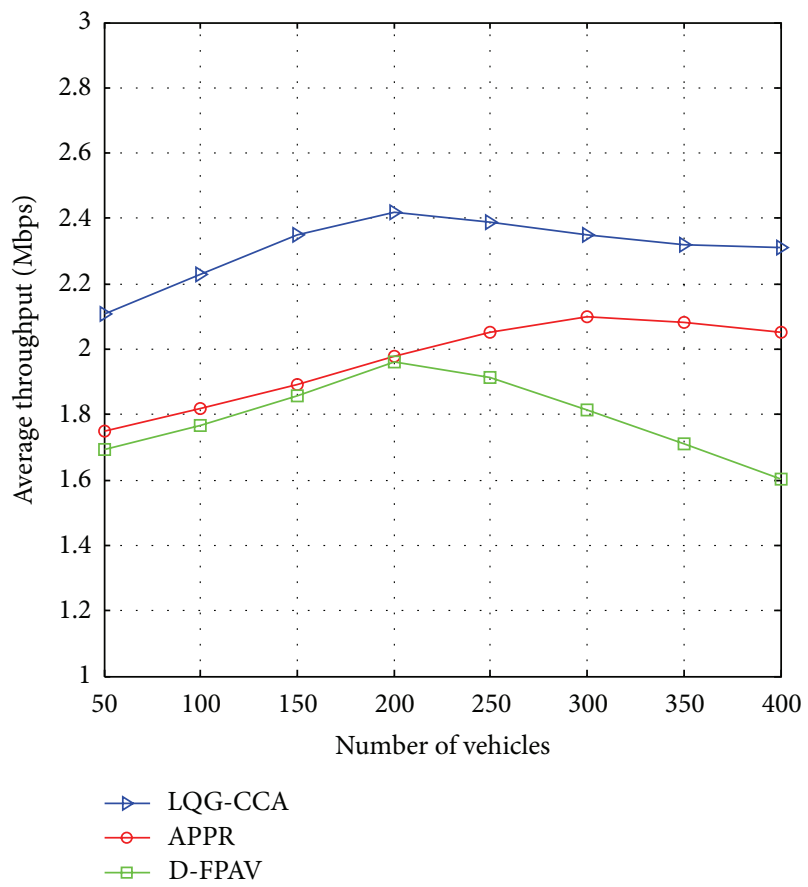

FIGURE 8: Average throughput versus number of vehicles when the packets generation speed is 5 packets/s.

CCA threshold for each active vehicle, the throughput could be maximized by forcing some vehicles to stop sending according to the channel load and contention level. In APPR, it also will stop the generation of some messages from vehicles if the packets loss ratio exceeds a predefined threshold. In this way, more transmission opportunities could be protected by suppressing extra interferences. However, in D-FPAV, the research emphasis falls on how to achieve the fairness of transmission opportunities among different active nodes. Every node starts with the minimum transmitting power assigned and then increases their transmitting power simultaneously with the same amount as long as the condition on the beaconing network load (MBL) is satisfied. In this 
way, the fairness is guaranteed but the total throughput in a network is damaged. As a result, D-FPAV outputs a lower average throughput when there are too many nodes simultaneously increasing their sending power, by which mutual interferences are also increased thus leading to a lower transmission opportunity for each node. In addition, the superiority of our LQG-CCA over APPR and D-FPAV is actually attributed to the distributed interference cancellation mechanism. In this way, the global throughput could be maximized by transmission opportunities assignment among nodes locally.

The performance of average E2E delay is shown in Figure 9 with the number of nodes varying from 50 to 400 . Note that the safety-related events are given the highest sending priority, that is, AC_VO, with smaller contention window and interframe space. It can be concluded that all three protocols meet the delay requirements for safety-related events according to "TABLE I" in [48], where a maximum $100 \mathrm{~ms}$ latency is required for both periodical and emergency warning messages. Since a larger density of vehicles will definitely increase the average E2E delay considering the packets collisions or transmission restraining, extra time is needed to finish the transmission attempts of active nodes. Our LQG-CCA also outperforms the other two protocols with a maximum latency approximately $5 \mathrm{~ms}$ at point 400 . The APPR ranks second while the D-FPAV is the worst. In addition, by checking the slope of three curves, it can be noticed that D-FPAV shows a faster growth of delay with the density increasing compared to the other two. This is actually because D-FPAV will easily saturate the channel load when there are too many nodes concurrently increasing their transmitting power. On the other hand, due to adaptive control of transmission opportunities or packet generation speed, LQG-CCA and APPR both show a more flat curve reflecting their better adaptability to the network load.

Figure 10 shows the average PDR of three algorithms with the number of nodes varying from 50 to 400 . Consistent with Figures 8 and 9, our LQG-CCA also ranks first considering its ability to adaptively adjust the medium sensing threshold thus leading to a better PDR. Note that the restrained nodes are not taken into account in the computation of PDR since they are no longer active. With the vehicular density increase, the average PDR of three protocols all drop. By checking the NS2 trace file, there are lots of "DROP_MAC_COLLISION", "DROP_MAC_BUSY", and "DROP_MAC_RETRY_COUNT_EXCEEDED" occurring; that is, the packets dropped due to collisions, channel being busy, and exceeding the retry limit, respectively. Therefore, we could say that the packets retrying and backing off contribute to the PDR falling when the density is increasing. However, since intelligent transmission scheduling is enabled in LQG-CCA and APPR, their PDR dropping is relatively slight with a minimum of 0.6 compared to D-FPAV which has a minimum PDR below 0.5. In addition, the smaller slope of LQG-CCA implies its better load adaptive capability compared to the other two.

The impact of packet generation speed on the average PDR is shown in Figure 11 with the packet generation speed varying from 0 to 10 packets/s. Note that, in this

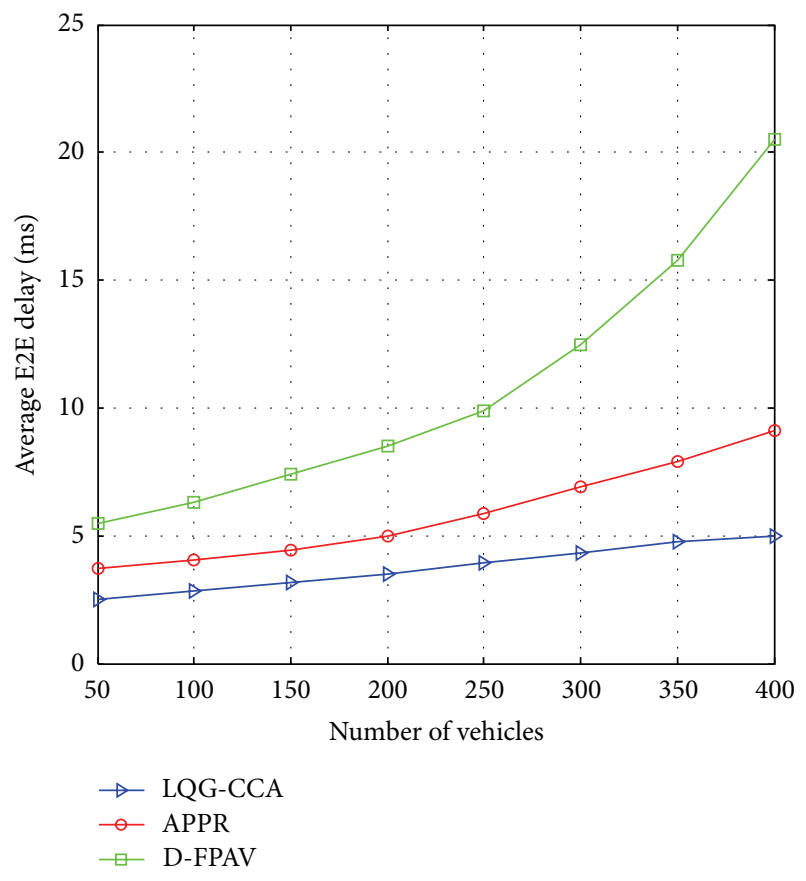

FIGURE 9: Average E2E delay versus number of vehicles when the packets generation speed is 5 packets/s.

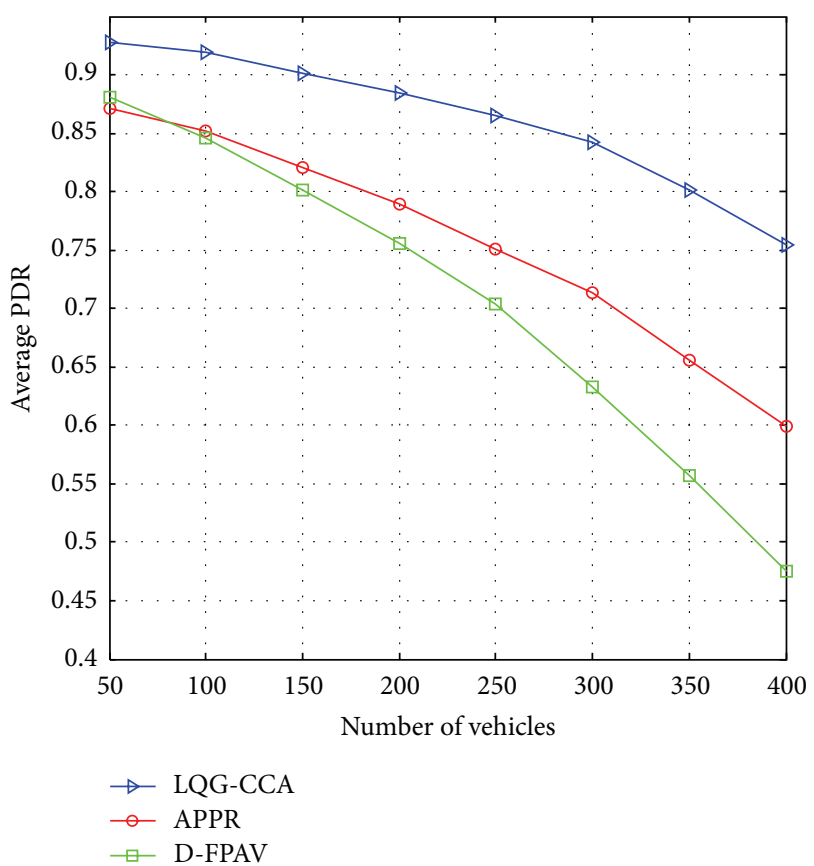

FIGURE 10: Average PDR versus number of vehicles when the packets generation speed is 5 packets/s.

simulation case, the number of nodes is fixed to 100 , say, a relatively slight density considering the total $10 \mathrm{~km}$ long road segment. With the packet generation speed increases, all three protocols show decreases of packets delivery ratio. This result is reasonable as a larger data sending rate will readily saturate the channel and bring more packets collisions thus 


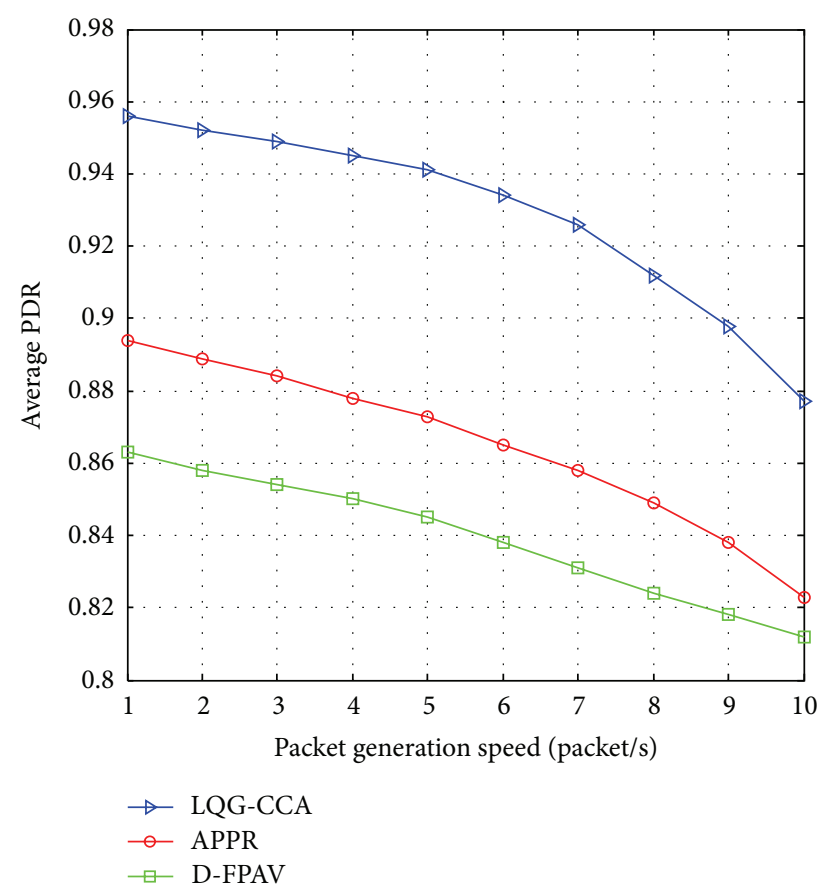

FIGURE 11: Average PDR versus packet generation speed when there are 100 nodes.

reducing the packets delivery ratio. What is noteworthy is that both LQG-CCA and APPR introduced the transmitting restraining scheme which will adaptively schedule the packets sending instead of just sending them out upon generation. As a result, although the packet generation speed is continuously increasing, the decreasing speed of three protocols is quite different according to their various medium access manners. For D-FPAV, there is no strategy to counteract the adverse impact on channel congestion from packet generation speed growing. Therefore, D-FPAV shows the worst performance consistent with previous numerical results. To compare APPR with our LQG-CCA, since there are no traffic predictions in APPR, their packet generation speed control mechanism will only take effect after the receiving of the feedback regarding a congestion that already occurred. In this way, our LQGCCA will act more efficiently with accurate estimations of the channel state thus leading to a better average PDR.

The impact of average intervehicle distance on the average PDR is shown in Figure 12. Note that although a 1000meter radio range is assumed, the packets are actually difficult to be received by their destinations 1000 meters away considering the channel fading and obstacles which are common in the urban environment such as skyscrapers, big trucks, and giant trees. As a matter of fact, the wireless signal will be attenuated by the equivalent walls according to our introduced obstacle model expressed by (31). To control the intervehicle distance, one parameter in IDM_LC, that is, the desired dynamical distance [49], has been changed to generate a required average intervehicle distance in the network. It is noteworthy that, with the increasing of average intervehicle distance, the average PDR drops quickly. This is because a larger intervehicle distance will bring more risks for

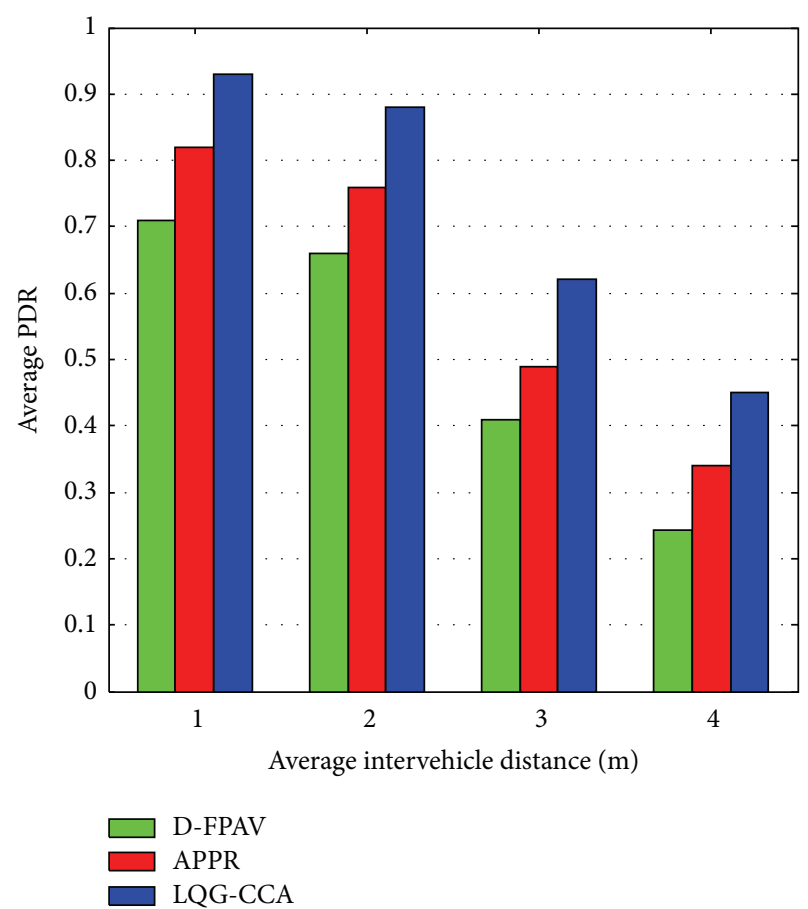

FIGURE 12: Average PDR versus average intervehicle distance when there are 100 nodes.

the signal to be sheltered by the obstacles. For our LQG-CCA, because its throughput maximization procedure considers the interferences from others using the SINR reception model, its performance degradation is the smallest compared to the other two. Since the reception model in APPR and D-FPAV is not given explicitly, we just determine whether a packet is successfully received according to the SNR threshold for frame reception listed in Table 3. However, due to the generation speed control according to the packet loss ratio in APPR, its performance is better than D-FPAV. As for D-FPAV, which puts its emphasis on access fairness rather than channel congestion and total throughput, it performs the worst among three models.

\section{Conclusion}

In this paper, we propose an LQG-based congestion control scheme in vehicular networks. Our model can dynamically adjust the CCA threshold according to the difference between the actual network throughput and the expected network throughput. In this way, the determined CCA threshold for each node can maximize the network throughput without leading to channel congestion at the same time. Numerical results show that our LQG optimized congestion control algorithm can improve the network throughput and packets delivery ratio and can reduce packet transmission delay significantly. Our future work will attempt to efficiently utilize the capture effect to make concurrent reception available on hardware and model the CCA threshold adjustable transmission model with MU-MIMO (Multiuser Multiple Input Multiple Output) enabled. 


\section{Nomenclature}

\begin{tabular}{|c|c|}
\hline$X(k)$ : & Predicted throughput \\
\hline A: & Coefficient matrix of the state variable \\
\hline$B:$ & $\begin{array}{l}\text { Coefficient matrix of the control } \\
\text { variable }\end{array}$ \\
\hline$U(k):$ & The control variable \\
\hline$W:$ & The system noise \\
\hline$Y(k):$ & The actual measured throughput \\
\hline$C:$ & $\begin{array}{l}\text { The coefficient matrix of measured } \\
\text { variable }\end{array}$ \\
\hline$V:$ & The measured noise \\
\hline Q: & The covariance of noise \\
\hline$R:$ & The weight of state variable \\
\hline$X(k \mid k-1):$ & The predicted throughput at time $k$ \\
\hline$X(k-1 \mid k-1):$ & The throughput at time $k-1$ \\
\hline$Z(k \mid k-1)$ & The covariance of predicted throughput \\
\hline$Z(k-1 \mid k-1)$ & $\begin{array}{l}\text { The covariance of throughput at time } \\
k-1\end{array}$ \\
\hline$\widehat{X(k)}:$ & The estimated throughput at time $k$ \\
\hline$Z(k \mid k):$ & The covariance of estimated throughput \\
\hline$Y_{r}(k):$ & The expected throughput \\
\hline$G(k):$ & The Kalman gain at time $k$ \\
\hline$H:$ & The covariance of measured noise \\
\hline$e(k)$ : & $\begin{array}{l}\text { The deviation of throughput from the } \\
\text { actual value }\end{array}$ \\
\hline$J:$ & $\begin{array}{l}\text { The objective function of quadratic } \\
\text { form }\end{array}$ \\
\hline$U^{*}(k):$ & The optimal control variable \\
\hline$S(k):$ & $\begin{array}{l}\text { The optimal output feedback gain } \\
\text { matrix. }\end{array}$ \\
\hline
\end{tabular}

\section{Notations}

$I_{s}: \quad$ The received signal strength

$P: \quad$ The transmission power

$r: \quad$ The distance between the transmitter and receiver

$\theta: \quad$ The path loss coefficient

$\mathrm{CCA}_{\text {th }}$ : The Clear Channel Assessment sensing threshold

$R: \quad$ The maximum transmission range

$W: \quad$ The channel bandwidth

$n$ : $\quad$ The number of concurrent transmissions

Area: The network range.

\section{Competing Interests}

The authors declare that they have no conflict of interests regarding the publication of this article.

\section{Acknowledgments}

This work was supported by the National Natural Science Foundation of China (61201133 and 61571338), the National Science and Technology Major Project of the Ministry of Science and Technology of China (2015zx03002006003), the Natural Science Foundation of Shaanxi Province (2014JM2-6089), the National High-Tech R\&D Program of
China (863 Program, 2015AA015701), the Research Collaboration Innovation Program of Xi'an (BD31015010001), the Hong Kong, Macao and Taiwan Science and Technology Cooperation Special Project (2014DFT10320 and 2015DFT10160), the Program for New Century Excellent Talents in University (NCET-11-0691), state grid cooperation project (HX0115014015), the Key Program of NSFCGuangdong Union Foundation (U1401251), and the "111 Project" of China (B08038).

\section{References}

[1] D. Gruyer, S. Demmel, B. D’Andrea-Novel, A. Lambert, and A. Rakotonirainy, "Simulation architecture for the design of cooperative collision warning systems," in Proceedings of the 15th International IEEE Conference on Intelligent Transportation Systems (ITSC '12), pp. 697-703, IEEE, Anchorage, Alaska, USA, September 2012.

[2] Y.-K. Park, Y.-J. Moon, Y.-S. Cho, and K.-J. Kum, "Field tests for evaluating cooperative intersection signal violation warning system (CISVWS)," International Journal of Automotive Technology, vol. 14, no. 2, pp. 275-281, 2013.

[3] L. Chen, S. H. Low, M. Chiang, and J. C. Doyle, "Cross-layer congestion control, routing and scheduling design in ad hoc wireless networks," in Proceedings of the 25th IEEE International Conference on Computer Communications (IEEE INFOCOM '06), pp. 1-13, Barcelona, Spain, April 2006.

[4] L. Chen, S. H. Low, and J. C. Doyle, "Joint congestion control and media access control design for ad hoc wireless networks," in Proceedings of the 24th Annual Joint Conference of the IEEE Computer and Communications Societies (IEEE INFOCOM '05), vol. 3, pp. 2212-2222, Miami, Fla, USA, March 2005.

[5] C. Lochert, B. Scheuermann, and M. Mauve, "A survey on congestion control for mobile ad hoc networks," Wireless Communications and Mobile Computing, vol. 7, no. 5, pp. 655-676, 2007.

[6] K. Kamini and R. Kumar, "VANET parameters and applications: a review," Global Journal of Computer Science and Technology, vol. 10, no. 7, pp. 72-77, 2010.

[7] J. Zhu, B. Metzler, X. Guo, and Y. Liu, "Adaptive CSMA for scalable network capacity in high-density WLAN: a hardware prototyping approach," in Proceedings of the INFOCOM 25th IEEE International Conference on Computer Communications, pp. 1-10, Barcelona, Spain, April 2006.

[8] F. Xia, H. Bin Liaqat, A. M. Ahmed et al., "User popularitybased packet scheduling for congestion control in ad-hoc social networks," Journal of Computer \& System Sciences, vol. 82, no. 1, pp. 93-112, 2016.

[9] W. Guan, J. He, L. Bai, and Z. Tang, "Adaptive congestion control of DSRC vehicle networks for collaborative road safety applications," in Proceedings of the 36th Annual IEEE Conference on Local Computer Networks (LCN '11), pp. 913-917, IEEE, Bonn, Germany, October 2011.

[10] S. Wang, L. Le, N. Zahariev, and K. K. Leung, "Centralized rate control mechanism for cellular-based vehicular networks," in Proceedings of the IEEE Global Communications Conference (GLOBECOM '13), pp. 4914-4920, IEEE, Atlanta, Ga, USA, December 2013.

[11] L. Wischhof and H. Rohling, "Congestion control in vehicular ad hoc networks," in Proceedings of the IEEE International Conference on Vehicular Electronics and Safety, pp. 58-63, October 2005. 
[12] M. S. Bouassida and M. Shawky, "A cooperative and fullydistributed congestion control approach within VANETs," in Proceedings of the 9th International Conference on Intelligent Transport Systems Telecommunications (ITST '09), pp. 526-531, IEEE, Lille, France, October 2009.

[13] L. Zhou, B. Zheng, B. Geller, A. Wei, S. Xu, and Y. Li, "Crosslayer rate control, medium access control and routing design in cooperative VANET," Computer Communications, vol. 31, no. 12, pp. 2870-2882, 2008.

[14] M. Torrent-Moreno, P. Santi, and H. Hartenstein, "Distributed fair transmit power adjustment for vehicular ad hoc networks," in Proceedings of the 3rd Annual IEEE Communications Society on Sensor and Ad hoc Communications and Networks (SECON '06), pp. 479-488, Reston, Va, USA, September 2006.

[15] W.-J. Guo, L.-S. Huang, Q. Sun, H.-L. Xu, and H.-R. Zhang, "Delay-aware reliable broadcast scheme based on power control for VANETs," The Journal of China Universities of Posts and Telecommunications, vol. 21, no. 1, pp. 26-35, 2014.

[16] S. Mitra and A. Mondal, "Joint congestion control strategy during V2V communication among authentic vehicles in VANET," Wireless Personal Communications, vol. 79, no. 1, pp. 43-67, 2014.

[17] C. Sommer, S. Joerer, M. Segata, O. K. Tonguz, R. L. Cigno, and F. Dressler, "How shadowing hurts vehicular communications and how dynamic beaconing can help," IEEE Transactions on Mobile Computing, vol. 14, no. 7, pp. 1411-1421, 2015.

[18] S. Bai, J. Oh, and J.-I. Jung, "Context awareness beacon scheduling scheme for congestion control in vehicle to vehicle safety communication," Ad Hoc Networks, vol. 11, no. 7, pp. 2049-2058, 2013.

[19] J. Sahoo, E. H.-K. Wu, P. K. Sahu, and M. Gerla, "Congestioncontrolled-coordinator-based MAC for safety-critical message transmission in VANETs," IEEE Transactions on Intelligent Transportation Systems, vol. 14, no. 3, pp. 1423-1437, 2013.

[20] N. Chaabouni, A. Hafid, and P. K. Sahu, "A collision-based beacon rate adaptation scheme (CBA) for VANETs," in Proceedings of the IEEE International Conference on Advanced Networks and Telecommunications Systems (ANTS '13), pp. 1-6, IEEE, Kattankulathur, India, December 2013.

[21] T. Tielert, D. Jiang, Q. Chen, L. Delgrossi, and H. Hartenstein, "Design methodology and evaluation of rate adaptation based congestion control for Vehicle Safety Communications," in Proceedings of the IEEE Vehicular Networking Conference (VNC '11), pp. 116-123, Amsterdam, The Netherlands, November 2011.

[22] M. A. Javed and J. Y. Khan, "Performance analysis of an adaptive rate-range control algorithm for VANET safety applications," in Proceedings of the International Conference on Computing, Networking and Communications (ICNC'14), pp. 418-423, IEEE, Honolulu, Hawaii, USA, February 2014.

[23] L. Zeng, L. Yang, Q. Han, X. He, L. Ye, and B. Yang, "Experimental analysis of CCA threshold self-adjusting method for EWM dissemination," in Proceedings of the 17th IEEE International Conference on Intelligent Transportation Systems (ITSC '14), pp. 3040-3045, Qingdao, China, October 2014.

[24] Q. Han, Y. Liu, L. Yang, and L. Zeng, "Experimental analysis of multi-hop vehicle node CCA threshold selection for EWM transmission," in Proceedings of the 2nd IEEE International Conference on Connected Vehicles and Expo (ICCVE '13), pp. 857-862, Las Vegas, Nev, USA, December 2013.

[25] B. Cho, K. Koufos, and R. Jantti, "Interference control in cognitive wireless networks by tuning the carrier sensing threshold," in Proceedings of the 8th International Conference on Cognitive Radio Oriented Wireless Networks and Communications (CROWNCOM '13), pp. 282-287, July 2013.

[26] R. K. Schmidt, A. Brakemeier, T. Leinmüller, F. Kargl, and G. Schäfer, "Advanced carrier sensing to resolve local channel congestion," in Proceedings of the 8th ACM International Workshop on Vehicular Inter-Networking (VANET '11), pp. 11-20, 2011.

[27] W. Li and E. Todorov, "Iterative linear quadratic regulator design for nonlinear biological movement systems," in Proceedings of the 1st International Conference on Informatics in Control, Automation and Robotics (ICINCO '04), pp. 222-229, Setúbal, Portugal, August 2004.

[28] C. L. Black, “The lawfulness of the segregation decisions," The Yale Law Journal, vol. 69, no. 3, pp. 421-430, 1960.

[29] L. Zhang, H. Gao, and O. Kaynak, "Network-induced constraints in networked control systems-a survey," IEEE Transactions on Industrial Informatics, vol. 9, no. 1, pp. 403-416, 2013.

[30] T. Schuhmann, W. Hofmann, and R. Werner, "Improving operational performance of active magnetic bearings using Kalman filter and state feedback control," IEEE Transactions on Industrial Electronics, vol. 59, no. 2, pp. 821-829, 2012.

[31] K. You, N. Xiao, and L. Xie, "LQG control with quantized innovation Kalman filter," in Analysis and Design of Networked Control Systems, pp. 205-221, Springer, London, UK, 2015.

[32] S. U. Eichler, "Performance evaluation of the IEEE 802.11p WAVE communication standard," in Proceedings of the IEEE 66th Vehicular Technology Conference (VTC-Fall '07), pp. 21992203, Baltimore, Md, USA, October 2007.

[33] X. Yang and N. Vaidya, "On physical carrier sensing in wireless ad hoc networks," in Proceedings of the IEEE 24th Annual Joint Conference of the IEEE Computer and Communications Societies, pp. 2525-2535, Miami, Fla, USA, March 2005.

[34] J. Q. Bao and L. Tong, "A performance comparison between ad hoc and centrally controlled CDMA wireless LANs," IEEE Transactions on Wireless Communications, vol. 1, no. 4, pp. 829841, 2002.

[35] D. P. Spanos, R. Olfati-Saber, and R. M. Murray, "Approximate distributed kalman filtering in sensor networks with quantifiable performance," in Proceedings of 4th International Symposium on Information Processing in Sensor Networks (IPSN '05), pp. 133-139, April 2005.

[36] G. Bianchi, "IEEE 802.11-saturation throughput analysis," IEEE Communications Letters, vol. 2, no. 12, pp. 318-320, 1998.

[37] V. A. Bavdekar, A. P. Deshpande, and S. C. Patwardhan, "Identification of process and measurement noise covariance for state and parameter estimation using extended Kalman filter," Journal of Process Control, vol. 21, no. 4, pp. 585-601, 2011.

[38] Z. Chao, S. Hongye, G. Yong, and C. Jian, "A pragmatic approach for assessing the economic performance of model predictive control systems and its industrial application," Chinese Journal of Chemical Engineering, vol. 17, no. 2, pp. 241-250, 2009.

[39] J. L. Willems and J. C. Willems, "Feedback stabilizability for stochastic systems with state and control dependent noise," Automatica, vol. 12, no. 3, pp. 277-283, 1976.

[40] H. Kwakernaak and R. Sivan, Linear Optimal Control Systems, vol. 1, Wiley-Interscience, New York, NY, USA, 1972.

[41] P. C. Young and J. C. Willems, "An approach to the linear multivariable servomechanism problem," International Journal of Control, vol. 15, pp. 961-979, 1972.

[42] J. Härri, F. Filali, C. Bonnet, and M. Fiore, "VanetMobiSim: generating realistic mobility patterns for VANETs," in Proceedings 
of the 3rd International Workshop on Vehicular ad hoc Networks (VANET '06), pp. 96-97, Los Angeles, Calif, USA, September 2007.

[43] C. Sommer, D. Eckhoff, R. German, and F. Dressler, "A computationally inexpensive empirical model of IEEE 802.11p radio shadowing in urban environments," in Proceedings of the 8th International Conference on Wireless On-Demand Network Systems and Services (WONS '11), pp. 84-90, Bardonecchia, Italy, January 2011.

[44] V. D. Khairnar and K. Kotecha, "Performance of vehicle-tovehicle communication using IEEE 802.11p in vehicular adhoc network environment," International Journal of Network Security \& Its Applications, vol. 5, no. 2, pp. 143-170, 2013.

[45] K. Bilstrup, E. Uhlemann, E. G. Strom, and U. Bilstrup, "Evaluation of the IEEE 802.11p MAC method for vehicle-to-vehicle communication," in Proceedings of the IEEE 68th Vehicular Technology Conference (VTC-Fall '08), pp. 1-5, Calgary, Canada, September 2008.

[46] V. Taliwal, D. Jiang, H. Mangold, C. Chen, and R. Sengupta, "Empirical determination of channel characteristics for DSRC vehicle-to-vehicle communication," in Proceedings of the 1st ACM International Workshop on Vehicular Ad Hoc Networks, p. 88, Philadelphia, Pa, USA, October 2004.

[47] S. Eichler, "Performance evaluation of the IEEE 802.11p WAVE communication standard," in Proceedings of the IEEE 66th Vehicular Technology Conference (VTC '07), pp. 2199-2203, IEEE, Baltimore, Md, USA, October 2007.

[48] E. C. Eze, S. Zhang, and E. Liu, "Vehicular ad hoc networks (VANETs): current state, challenges, potentials and way forward," in Proceedings of the 20th International Conference on Automation and Computing (ICAC '14), pp. 176-181, Cranfield, UK, September 2014.

[49] M. Fiore, J. Harri, F. Filali, and C. Bonnet, "Vehicular mobility simulation for VANETs," in Proceedings of the 40th Annual Simulation Symposium (ANSS '07), pp. 301-309, Norfolk, Va, USA, March 2006. 

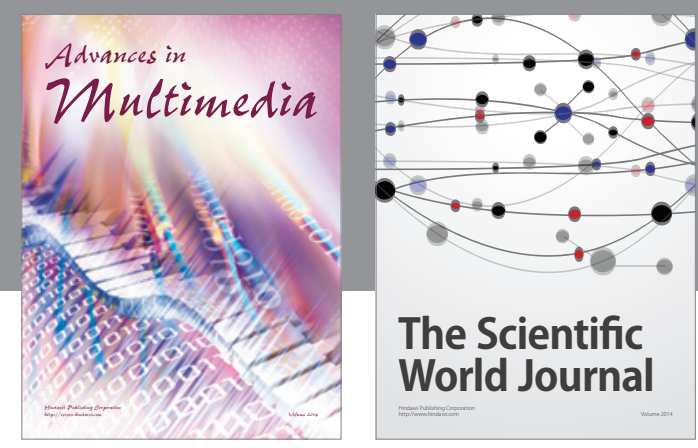

The Scientific World Journal
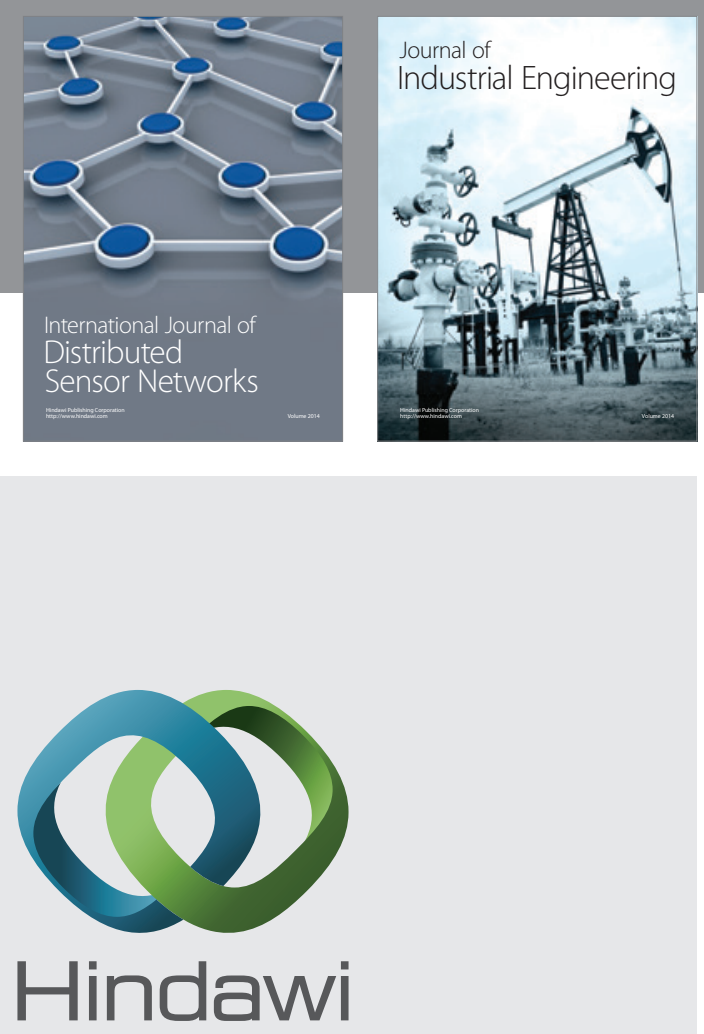

Submit your manuscripts at

http://www.hindawi.com

\section{Computer Networks} and Communications
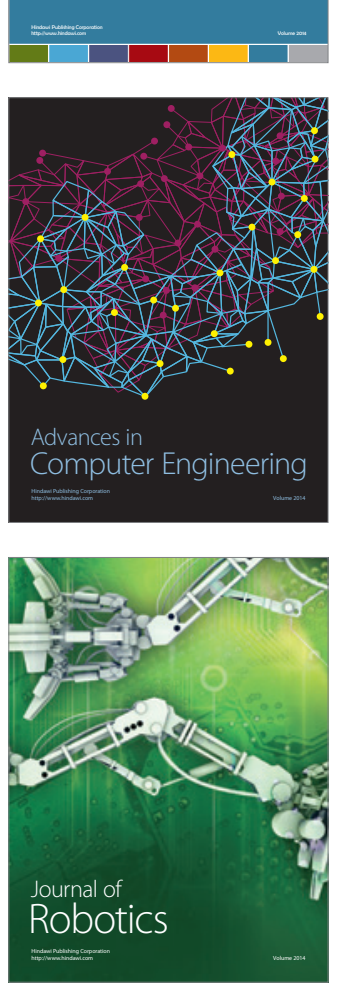
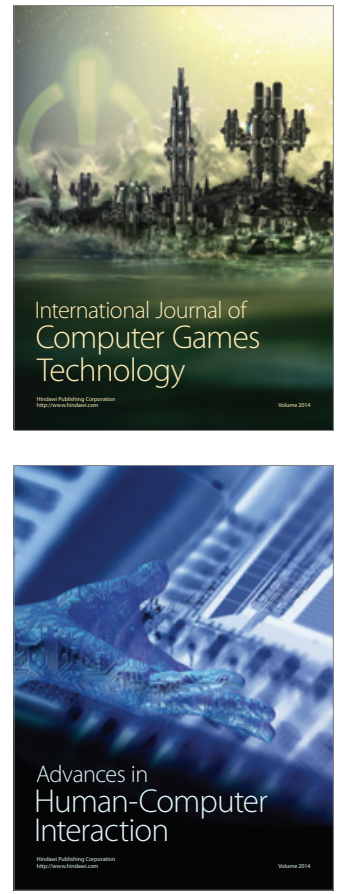
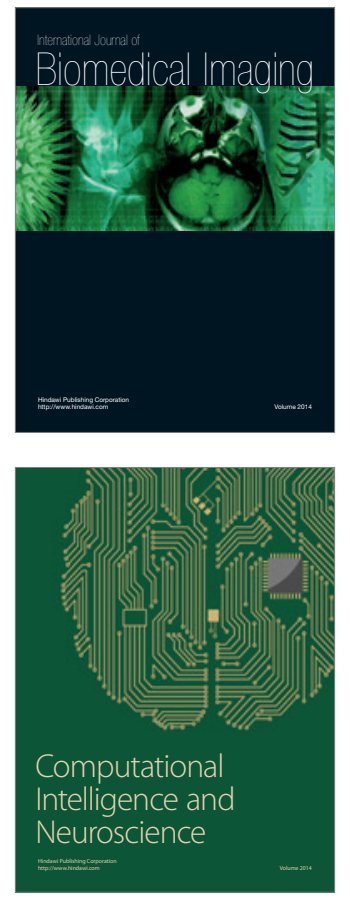
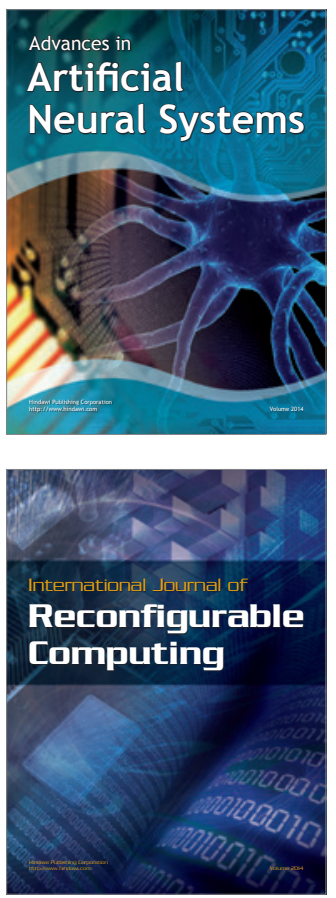
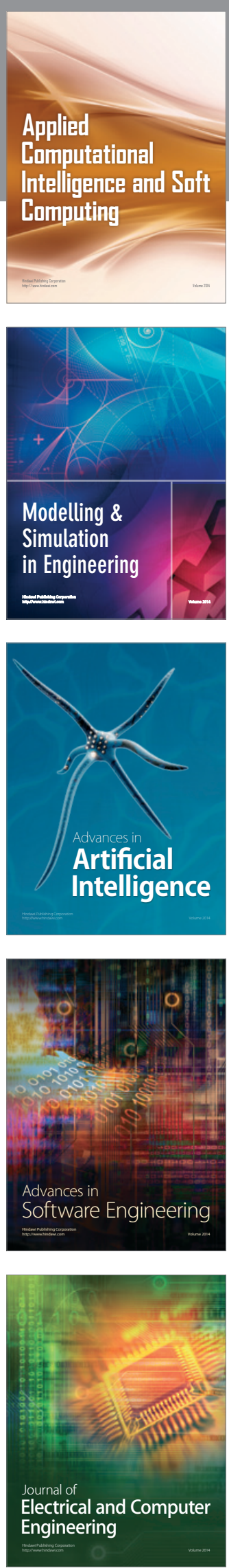